\title{
ISOFLAVONOIDES DA TRIBO DALBERGIEAE: UMA CONTRIBUIÇÃO QUIMIOSSISTEMÁTICA PARA A SUBFAMÍLIA PAPILIONOIDEAE
}

\author{
Adonias A. Carvalho, , Lucivania R. dos Santosa, Jurema S. de Freitas e Mariana H. Chaves,*,() \\ aDepartamento de Química, Universidade Federal do Piauí, 64049-550 Teresina - PI, Brasil \\ bDiretoria de Ensino, Instituto Federal do Piauí, 64260-000 Piripiri - PI, Brasil
}

Recebido em 28/02/2020; aceito em 25/05/2020; publicado na web em 08/07/2020

\begin{abstract}
ISOFLAVONOIDS OF THE TRIBE DALBERGIEAE: A CHEMOSYSTEMATIC CONTRIBUTION TO THE SUBFAMILY PAPILIONOIDEAE. Plants from Fabaceae family have several secondary metabolites and high biological potential. The subfamily Papilionoideae (Fabaceae) has 28 tribes, of which Dalbergieae comprises 49 genera and 1325 species. Isoflavonoids are chemotaxonomic markers of Papilionoideae and have important biological properties. This review describes the isoflavonoids and biological activities from the tribe Dalbergieae (Fabaceae-Papilionoideae) species, in the period of 1945 to 2019. A total of 240 isoflavonoids were found in 69 species and in 15 genera. Formononetin, biochanin A and medicarpin were the most frequent isoflavonoids in this tribe. Dalbergia, Machaerium, Andira and Pterocarpus genera had a high number of species with occurrence of isoflavonoids. A total of 81 isoflavanoids and 46 species showed biological activities. This suggests that more works are necessary to evaluate the potential of this tribe. The present study contributes to the taxonomic classification of genus or species in the family, subfamily or tribe, once the correlation between secondary metabolites and morphological data is an important tool for the classification, phylogeny and evolution of species. This compilation also contributes to extend and update the researched period on the occurrence of isoflavonoids in species of the tribe Dalbergieae, described in the literature in other bibliographic reviews.
\end{abstract}

Keywords: isoflavonoids; isoflavones; Dalbergieae; Papilionoideae; Fabaceae.

\section{INTRODUÇÃO}

Estudos quimiossistemáticos ou quimiotaxonômicos utilizam dados químicos provenientes do metabolismo especializado ou micromolecular das plantas e podem auxiliar na identificação e classificação de espécies de difícil caracterização com o uso somente de análises morfológicas. A quimiossistemática considera o fato dos metabólitos especializados e suas vias biossintéticas serem frequentemente específicas e restritas a organismos taxonomicamente relacionados. ${ }^{1,2}$

A grande variedade de constituintes químicos isolados de espécies vegetais em conjunto com a morfologia e dados citológicos permitem diagnosticar o histórico do organismo e as modificações sofridas em seu ambiente. ${ }^{3}$

Recentemente, um novo termo denominado quimiofenética foi proposto para descrever estudos quimiossistemáticos que não visem somente elucidar relações filogenéticas, mas também descrever a variedade de produtos naturais micro e macromoleculares de um determinado táxon. ${ }^{1,4}$ Dessa forma, além das abordagens anatômicas, morfológicas e cariológicas já reconhecidas como de grande importância para o estabelecimento de sistemas naturais, os estudos quimiofenéticos reúnem uma combinação de dados fitoquímicos e macromoleculares e até mesmo a busca sistemática de produtos naturais raros que podem ajudar na caracterização de clados, até agora suportados apenas por dados de sequência de DNA. ${ }^{4}$

Plantas da família Fabaceae Lind (Leguminosae) são conhecidas pela diversidade de metabólitos secundários e por seu elevado potencial biológico. ${ }^{5} \mathrm{O}$ modo de distribuição destes metabólitos pode ser usado para identificar a origem botânica e muitas vezes expressa a adaptação, regulação e evolução sofrida por um determinado táxon. $\cdot^{6-8}$

Os isoflavonoides são metabólitos secundários ou micromoleculares quase que exclusivamente restritos a subfamília Papilionoideae

*e-mail: mariana@ufpi.edu.br
(Fabaceae), sendo alvo de três revisões bibliográficas compreendendo o período de 1997 a $2011 .{ }^{6}$ Entretanto, considerando a relevância dos metabólitos secundários como instrumentos de classificação taxonômica, que a tribo Dalbergieae (Fabaceae-Papilionoideae) passou por várias modificações, especialmente, após avanços na filogenia molecular, ${ }^{9}$ o presente trabalho teve como objetivo realizar uma revisão bibliográfica dos isoflavonoides e atividades biológicas de espécies desta tribo, no período de 1945 a 2019, contribuindo para ampliar e atualizar o período pesquisado sobre a ocorrência destes metabólitos nesta tribo, como também ajudar na caracterização de clados e fornecer subsídios para guiar futuras prospeções fitoquímicas que visem a descoberta de novos agentes terapêuticos.

\section{O táxon Fabaceae}

Fabaceae é considerada a terceira maior família de Angiospermas em número de espécies ficando atrás apenas de Asteraceae e Orchidaceae. É constituída por aproximadamente 36 tribos, 770 gêneros, 19.500 espécies e está distribuída em quase todos os continentes, exceto na Antártida. ${ }^{7,10}$ Essa família é a segunda em importância econômica, ficando atrás apenas da Poaceae. ${ }^{7,9}$

No Brasil, Fabaceae é constituída por 213 gêneros, 2.756 espécies, sendo 1.458 endêmicas, 53 subespécies e 731 variedades. ${ }^{11}$ A classificação tradicional dividia a família Fabaceae em três subfamílias: Papilionoideae, Caesalpiniodeae e Mimosoideae, as quais podem ser diferenciadas principalmente pela prefloração das pétalas. ${ }^{10}$ No entanto, uma classificação mais recente baseada em estudos taxonômicos e moleculares foi endossada pelo Legume Phylogeny Working Group, ${ }^{7}$ no qual dividiu a família Fabaceae em seis subfamílias: Papilionoideae (503 gêneros, cerca 14.000 espécies), Caesalpinioideae (148 gêneros, cerca de 4.400 espécies; incluindo os gêneros do clado Mimosoideae), Detarioideae (84 gêneros, cerca de 760 espécies), Dialioideae (17 gêneros, cerca 85 espécies), Cercidoideae (12 gêneros, cerca de 335 espécies) e Duparquetioideae (1 gênero, 1 espécie). ${ }^{7}$ 
Plantas pertencentes a Fabaceae são ricas em flavonoides, porém acumulam também outros metabólitos como alcaloides, terpenoides e esteroides. ${ }^{6,12,13}$ Devido sua ampla distribuição global e sua inegável importância em diversos biomas, as espécies de Fabaceae estão presentes na vida humana há milênios e são extremamente importantes do ponto de vista econômico. ${ }^{14}$ A contribuição na alimentação humana e de outros animais permitem que diversas indústrias sejam beneficiadas pelos produtos derivados de Fabaceae, como medicamentos, corantes, pesticidas, resinas, tintas, gomas e espessantes. Espécies dessa família também vêm sendo usadas para a melhoria de solos agrícolas, em função de sua grande capacidade de fixação de nitrogênio gerada pela atividade de bactérias simbiontes existentes nos nódulos radiculares. ${ }^{14,15}$ Muitas espécies são também utilizadas em ornamentação e paisagismo e outras são ainda organismos modelo para diversos tipos de estudo. ${ }^{10}$ No Brasil, é a principal família utilizada para a arborização urbana e várias de suas espécies são usadas na indústria madeireira, por fornecerem matéria prima de alta qualidade. ${ }^{15}$

\section{Subfamília Papilionoideae - Tribo Dalbergieae}

Papilionoideae constitui a maior subfamília de Fabaceae, distinguindo-se das outras subfamílias vegetativamente e pelos caracteres florais e do fruto, sendo considerada monofilética. ${ }^{716}$ Essa subfamília atualmente está representada por 28 tribos, das quais Dalbergieae compreende 49 gêneros e cerca de 1.325 espécies, com cinco gêneros pantropicais, um anfiatlântico e dois transatlânticos. ${ }^{10}$ Essa tribo apresenta grande diversidade na América do Sul com 38 gêneros e, no Brasil, são relatados a ocorrência de $28 .{ }^{16}$ Estudos filogenéticos mostram os grandes clados em que a tribo Dalbergieae está dividida: clado Adesmia, composto por 6 gêneros e cerca de 360 espécies, de distribuição principalmente neotropical; clado Pterocarpus, incluindo 22 gêneros e cerca de 200 espécies, centrado na região neotropical, com alguns componentes se expandindo para a região Pantropical; clado Dalbergia, com 17 gêneros e cerca de 700 espécies, de distribuição pantropical, centrado principalmente na África e por fim os quatro gêneros isolados Andira, Hymenolobium, Vatairea e Vataireopsis que reúnem 58 espécies de distribuição majoritariamente neotropical. Desta forma, explica-se a interessante composição atual da tribo Dalbergieae sensu lato, com representantes diversos e aparentemente bastante distantes, porém intimamente relacionados..$^{9,16,17}$

\section{Isoflavonoides}

Os flavonoides são metabólitos secundários sintetizados por plantas e apresentam, de modo geral em sua estrutura, várias hidroxilas ligadas a anel aromático, por isso são chamados de compostos polifenólicos. O esqueleto básico de compostos dessa classe é oriundo de rota biossintética mista (Figura 1), sendo formado por 15 átomos de carbono dispostos em dois anéis aromáticos conectados por uma ponte de três átomos de carbono $\mathrm{C}_{6}-\mathrm{C}_{3}-\mathrm{C}_{6} \cdot{ }^{8}{ }^{818}$ Estima-se a existência de cerca de 5.000 estruturas conhecidas de flavonoides que estão agrupadas, baseadas no estado oxidativo do anel C, em oito subclasses: flavanois, flavandiois, flavanonas, di-hidroflavanois, flavonas, flavonois, antocianidinas e isoflavonoides. ${ }^{8}$

A subclasse isoflavonoides possui cerca de 1.000 estruturas conhecidas, é encontrada em uma grande variedade de espécies de Papilionoideae, sendo considerada marcadores quimiotaxonômicos desta subfamília. Diferentemente de outros flavonoides, os isoflavonoides apresentam o anel B ligado na posição C-3 em vez de C-2, além de sofrer várias modificações estruturais que geram isoflavonoides simples, como isoflavonas, isoflavanonas, isoflavanas e isoflavanois (isoflavan-4-ol), bem como estruturas mais complexas, incluindo rotenoides, pterocarpanos e cumestanos (Figura 1). ${ }^{8,18}$
Os estudos iniciais com isoflavonoides concentravam-se em suas propriedades como marcadores quimiossistemáticos. Com o passar do tempo tornaram-se conhecidos por suas propriedades antifúngicas, conforme relatado para medicarpina (156) e vestitol (195), em alguns casos por fazerem parte dos mecanismos de defesa da planta e inseticidas, como também por serem considerados fitoalexinas por fitofisiologistas. ${ }^{18,19}$ As isoflavonas estão associadas a muitas atividades biológicas, incluindo tratamento da osteoporose, doenças cardiovasculares, sintomas da menopausa e prevenção do câncer. ${ }^{13}$ Estudos indicam que o efeito protetor dos isoflavonoides pode se estender além de suas atividades antioxidantes em níveis moleculares e celulares. ${ }^{8,18}$

A estrutura química das isoflavonas é muito semelhante à do hormônio estrogênico animal (estradiol e testosterona), de modo que alguns compostos dessa classe possuem atividade estrogênica. ${ }^{20} \mathrm{O}$ consumo de isoflavonoides é estimulado na dieta humana, visando a proteção contra cânceres dependentes de estrogênio (câncer de mama), quando a disponibilidade do hormônio natural é restrita. Dessa forma, são empregados como suplementos dietéticos de estrogênio para a redução dos sintomas da menopausa, de forma semelhante à terapia de reposição hormonal. ${ }^{18}$

\section{ISOFLAVONOIDES DA TRIBO DALBERGIEAE}

A presente revisão bibliográfica consistiu na ampliação e atualização do período pesquisado em outras revisões ${ }^{6}$ sobre a ocorrência dos isoflavonoides e suas propriedades biológicas relatados em espécies da tribo Dalbergieae (Fabaeceae-Papilionoideae), contribuindo para uma melhor compreensão do metabolismo micromolecular e sua ação no organismo animal, bem como ajudar na caracterização de clados e guiar futuros estudos fitoquímicos que visem a descoberta de novos agentes terapêuticos.

No período de 1945 a 2019 foram relatados 240 isoflavonoides na tribo Dalbergieae, distribuídos em 15 gêneros e 69 espécies (Tabela 1). Os isoflavonoides foram relacionados por classes: isoflavonas (1 a $\mathbf{1 2 5}, \mathbf{5 2 , 1 \%})$, isoflavanonas (126 a $\mathbf{1 5 5}, \mathbf{1 2 , 5 \% )}$ ), pterocarpanos (156 a $\mathbf{1 8 2}, \mathbf{1 1}, 2 \%)$, rotenoides $(\mathbf{1 8 3}$ a $\mathbf{1 9 4}, 5,0 \%)$, isoflavanas, isoflavanquinonas e isoflavan-4-ois (195 a $\mathbf{2 2 5}, 13,0 \%)$ e outros isoflavonoides (226 a 240, 6,2\%). Os isoflavonoides glicosilados totalizaram 48 (20\%). Os compostos de maior ocorrência são as isoflavonas formononetina (2) e biochanina A (17) e o pterocarpano medicarpina (156) presentes em 20, 19 e 16 espécies, respectivamente. Os gêneros que apresentaram maior número de espécies com ocorrência de isoflavonoides foram Dalbergia (31 espécies), Machaerium (9 espécies), Andira (6 espécies) e Pterocarpus (6 espécies). A revisão bibliográfica mostrou ainda, três trabalhos que identificaram por Cromatografia Líquida de Alta Eficiência acoplada a Espectrometria de Massas (CLAE-EM) 35 isoflavonoides, dos quais 12 eram isoflavonas, 13 isoflavanonas, 8 isoflavanas e 2 pterocarpanos. Estes compostos foram previamente isolados e relatados em outros trabalhos. ${ }^{20-22}$

Dalbergia parviflora apresentou o maior número de isoflavonoides, com 46 compostos isolados. Essa espécie é conhecida na Tailândia como "sak kee", onde é utilizada na medicina tradicional como expectorante, estomacal, cardiotônico e para regular o ciclo menstrual. ${ }^{20,23}$ As folhas desta planta têm efeito afrodisíaco suave e o óleo do cerne (vermelho-escuro) é usado como antipirético e no tratamento de feridas crônicas. ${ }^{20}$ Devido a essas propriedades, essa espécie tem sido alvo de estudos químicos e biológicos. ${ }^{23}$

\section{Isoflavonas}

Isoflavonas estão presentes na natureza predominantemente como $\beta$-glicosídeos, acetil- $\beta$-glicosídeos e malonil- $\beta$-glicosídeos, todos solúveis em água. Pequenas modificações estruturais produzem 

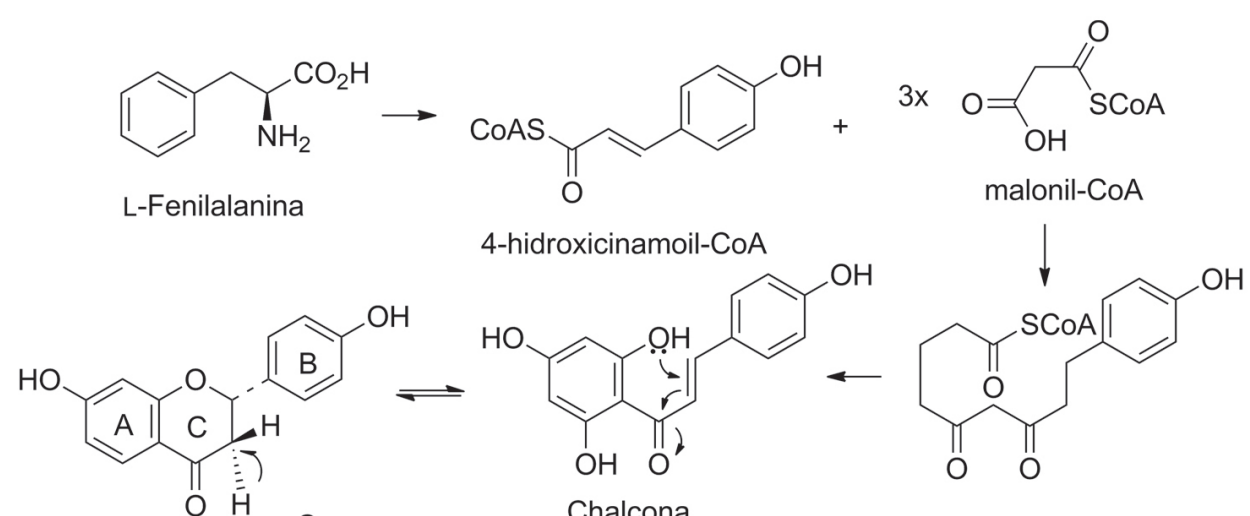

Flavanona $\mathrm{O}$

Chalcona

(liquiritigenina) $\mathrm{Fe}-\mathrm{Enz}$

$\mathrm{O}_{2}$

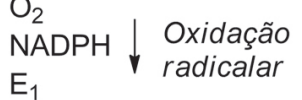

$\mathrm{E}_{1}$

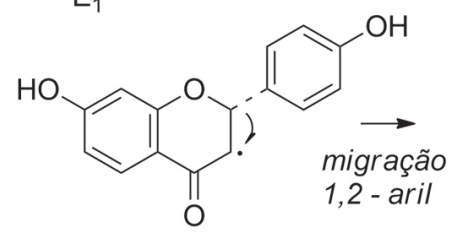

$\mathrm{HO}$<smiles>O=C1Oc2ccccc2C(=O)C1c1ccc(O)cc1</smiles><smiles>O=C1c2ccc(O)cc2OC(O)C1c1ccc(O)cc1</smiles>

$E_{3} \downarrow-\mathrm{H}_{2} \mathrm{O}$

$E_{1}$ : 2-hidroxiisoflavanona sintase

$E_{2}: 2,7,4$ '-trihidroxiisoflavanona/4'-O-metiltransferase

$E_{3}, E_{4}:$ 2-hidroxiisoflavanona dehidratase

$\mathrm{E}_{2}$<smiles>C=C(C)[C@H]1Cc2c(ccc3c2O[C@H]2COc4cc(OC)c(OC)cc4[C@H]2C3=O)O1</smiles><smiles>COc1ccc(C2C(=O)c3ccc(O)cc3OC2O)cc1</smiles>

$\mathrm{E}_{4} \downarrow-\mathrm{H}_{2} \mathrm{O}$<smiles>O=c1c(-c2ccc(O)cc2)coc2cc(O)ccc12</smiles>

Isoflavona

(daidzeina)<smiles>COc1ccc(C2COc3cc(O)ccc3C2=O)cc1</smiles>

$\mathrm{HO}$<smiles>COc1ccc(-c2coc3cc(C)ccc3c2=O)cc1</smiles>

Isoflavona (formononetina)<smiles>COc1ccc(C2COc3cc(O)ccc3C2O)cc1</smiles>

Isoflavanol

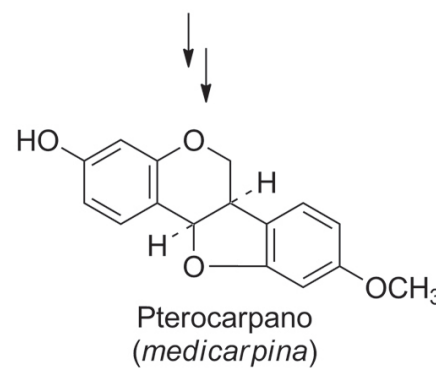<smiles>O=c1oc2cc(O)ccc2c2c1oc1cc(O)ccc12</smiles>

Coumestano (coumestrol)<smiles>COc1ccc([C@H]2COc3cc(O)ccc3C2)c(O)c1</smiles>

Figura 1. Biossíntese de isoflavonoides, adaptada da ref. 18

significativas alterações em suas propriedades biológicas e bioquímicas. Uma das propriedades químicas mais importantes das isoflavonas é a capacidade de participar de processos redox..$^{8,140}$

As isoflavonas dietéticas podem ser classificadas em quatro categorias: (I) agliconas (sem ligação com açúcar): daidzeína (1), formononetina (2), genisteína (16), biochanina A (17) e gliciteina (33); (II) glicosídeos ou gliconas: daidzina, genistina, glicitina, ononina (5) e sissotrina; (III) acetilglicosídeos ou acetilgliconas: 6"- acetildaldazina, 6"-acetilgenistina e 6"-acetilglicitina; (IV) malonilglicosídeos ou malonilgliconas: 6"-malonilaldazina, 6"-malonilgenistina e 6"-malonilglicitina. ${ }^{8}$

O número de isoflavonas encontrado na literatura excede o de qualquer outra subclasse de isoflavonoides. ${ }^{8} \mathrm{Na}$ tribo Dalbergieae as isoflavonas são a subclasse de isoflavonoides mais frequentes com 
Tabela 1. Isoflavonoides de espécies da tribo Dalbergieae

\begin{tabular}{|c|c|c|c|c|}
\hline Gênero & Espécie & Parte da planta & Compostos & Ref. \\
\hline \multirow{4}{*}{ Aeschynomene } & \multirow{2}{*}{ A. fascicularis } & casca das raízes & Isoflavana: 217 & 24 \\
\hline & & casca das raízes & Pterocarpanos: 168 e 182 & 25 \\
\hline & A. fluminensis & folhas e galhos & Isoflavona: 1 & 26 \\
\hline & A. sensitiva & partes aéreas e sementes & Isoflavona: 47; Rotenoide: 183 & 27 \\
\hline \multirow{8}{*}{ Andira } & A. anthelmia & raízes & Isoflavonas: $16,17,22,27$ e 28 & 28 \\
\hline & A. fraxinifolia & raízes & Isoflavonas: $\mathbf{1 7}$ e $\mathbf{5 0}$ & 29 \\
\hline & A. humilis & raízes & Isoflavonas: 16, 17, 22, 25, 26, 27 e 50; Isoflavanona: 126 & 30 \\
\hline & \multirow{3}{*}{ A. inermis } & folhas & $\begin{array}{l}\text { Isoflavonas: } 2,10,18 \text { e 32; 2-Arilbenzofurano-3-carbaldeídos: } \\
\text { 236, } 237 \text { e } 238\end{array}$ & 31 \\
\hline & & casca do caule e sementes & Isoflavonas: $\mathbf{2}, \mathbf{1 0}, \mathbf{1 6}, \mathbf{1 7}, \mathbf{1 8}$ e $\mathbf{5 0}$ & 32 \\
\hline & & raízes & Isoflavonas: $\mathbf{2 1}, \mathbf{2 6}$ e 27 & 33 \\
\hline & A. parviflora & cerne & Isoflavonas: 16 e 17; Isoflavana: 202 & 34 \\
\hline & A. surinamensis & casca dos galhos & Isoflavonas: $10,17,18$ e 50 & 35 \\
\hline Arachis & A. hypogaea & sementes & Pterocarpanos: 174 e 178 & 36 \\
\hline Brya & B. ebenus & madeira de cocus & $\begin{array}{l}\text { Isoflavanas: 203, 204, } 205 \text { e 212; Pterocarpenos: 228, 229, 230, } \\
231 \text { e } 232\end{array}$ & 37 \\
\hline \multirow[b]{2}{*}{ Centrolobium } & C. sclerophyllum & cerne & Isoflavona: 10 & 38 \\
\hline & $\begin{array}{c}\text { C. sclerophyllum; } C \text {. } \\
\text { paraense; C. tomentosum e } \\
\text { C. robustum }\end{array}$ & cerne & $\begin{array}{l}\text { Isoflavonas: } 2 \text { e 10; Isoflavanona: } 139 \text {; Pterocarpano: 156; Isofla- } \\
\text { vanas: 195, } 198 \text { e 200; Isoflaveno: } 226\end{array}$ & 39 \\
\hline \multirow{30}{*}{ Dalbergia } & D. boehmii & cerne & $\begin{array}{l}\text { Isoflavonas: 14, 16, } 17 \text { e 44; Pterocarpanos: } 156,157 \text { e } 158 \text {; Cou- } \\
\text { mestano: 233; Coumaronocromona: } 239 ;\end{array}$ & 40 \\
\hline & \multirow{2}{*}{ D. candenatensis } & cerne & Isoflavonas: 2 e 107; Isoflavanas: 195 e 200; Isoflavanquinona: 222 & 41 \\
\hline & & cerne & Pterocarpanos: 179 e 180; Isoflavana: 199 & 42 \\
\hline & D. cochinchinensis & sementes & Rotenoides: 184, 186 e 187 & 43 \\
\hline & D. congesta & raízes & Isoflavonas: $\mathbf{8 5}$ & 44 \\
\hline & D. coromandeliana & folhas & Isoflavona: $\mathbf{2 0}$ & 45 \\
\hline & \multirow{3}{*}{ D. ecastaphyllum } & casca do caule & asoflavonas: 1, 2 e 17 & 21 \\
\hline & & cerne & $\begin{array}{l}\text { Isoflavonas: } 2,13 \text { e } 17 ; \text { Pterocarpanos: } 156,157,160,162,163 \text { e } \\
\text { 165; Isoflavanas: } 195,196,197 \text { e } \mathbf{2 0 0}\end{array}$ & 46 \\
\hline & & cerne & Isoflavona: 2; Isoflavanas: 200 e 205 & 47 \\
\hline & D. frutescens & casca do caule & Isoflavonas: $\mathbf{1}, \mathbf{2}, 7, \mathbf{1 6}, \mathbf{1 7}, \mathbf{3 2}, \mathbf{3 3}, \mathbf{3 4}, 35$ e 69 & 48 \\
\hline & D. horrida & raízes & Isoflavonas: 85 e 95; Isoflavanonas: 140 e 152 & 49 \\
\hline & D. lanceolaria & casca das raízes & Isoflavona: $\mathbf{2 6}$ & 50 \\
\hline & D. melanoxylon & casca do caule & Isoflavona: 2; Isoflavononas: 151 e 153 & 51 \\
\hline & \multirow{2}{*}{ D. monetaria } & casca do caule & Isoflavonas: 49, 103, 106, 111, 112, 116 e 121 & 52 \\
\hline & & sementes & Isoflavonas: 45 e 46; Rotenoides: 184, 187, 188, 189, 190, 191 e 192 & 53 \\
\hline & D. nigra & folhas secas & Isoflavona: 67 & 54 \\
\hline & \multirow{2}{*}{ D. nitidula } & cerne & Isoflavona: 42 & 55 \\
\hline & & cerne & Pterocarpanos: 164, 171 e 172 & 56 \\
\hline & \multirow{12}{*}{ D. odorifera } & folhas & Isoflavonas: 16, 17, 18 e 64 & 57 \\
\hline & & cerne & Isoflavononas: 128, 129 e 141; Isoflavana: 195 & 58 \\
\hline & & cerne & $\begin{array}{l}\text { Isoflavonas: } 1,2,49,91 \text { e } 106 \text {; Isoflavononas: } 85,129,132,133 \text {, } \\
136 \text { e 141; Pterocarpano: } 156\end{array}$ & 59 \\
\hline & & folhas & Isoflavonas: 16 e 17 & 60 \\
\hline & & cerne & $\begin{array}{l}\text { asoflavonas: } 2,13,15,70 \text { e } 95 \text {; Isoflavanonas: 128, 129, 133, } 135 \\
\text { e 141; Pterocarpanos: } 156 \text { e 166; Isoflavanquinona: } 222\end{array}$ & 22 \\
\hline & & cerne & $\begin{array}{l}\text { Isoflavonas: } 2 \text { e 64; Pterocarpano: 156; Isoflavanas: } 200 \text { e } \mathbf{2 0 1} \text {; } \\
\text { Isoflavanquinona: } 222\end{array}$ & 61 \\
\hline & & cerne & Isoflavona: 2; Pterocarpano: 156; Isoflavanquinona: 222 & 62 \\
\hline & & cerne & $\begin{array}{l}\text { Isoflavonas: } 2,9,15,18,44 \text { e } 70 ; \text { Isoflavononas: } 128,129,133,135 \\
\text { e 141; Pterocarpano: } 156\end{array}$ & 63 \\
\hline & & cerne & $\begin{array}{l}\text { Isoflavona: 2; Pterocarpanos: 156, 166, 175, } 176 \text { e 177; Isoflavanas: } \\
\text { 195, 200, } 208 \text { e 209; Isoflavena: } 227\end{array}$ & 64 \\
\hline & & cerne & Isoflavonas: 2 e 11; Isoflavanas: 195 e 201; Isoflavanquinona: 222 & 65 \\
\hline & & cerne & $\begin{array}{l}\text { Isoflavonas: 2, } 11 \text { e 48; Isoflavanona: 141; Pterocarpano: 156; } \\
\text { Isoflavanas: 195, } 201 \text { e 206; Isoflavanquinona: } 222\end{array}$ & 66 \\
\hline & & cerne & Isoflavona: 2; Isoflavonona: 133; Pterocarpano: 156 & 67 \\
\hline
\end{tabular}


Tabela 1. Isoflavonoides de espécies da tribo Dalbergieae (cont.)

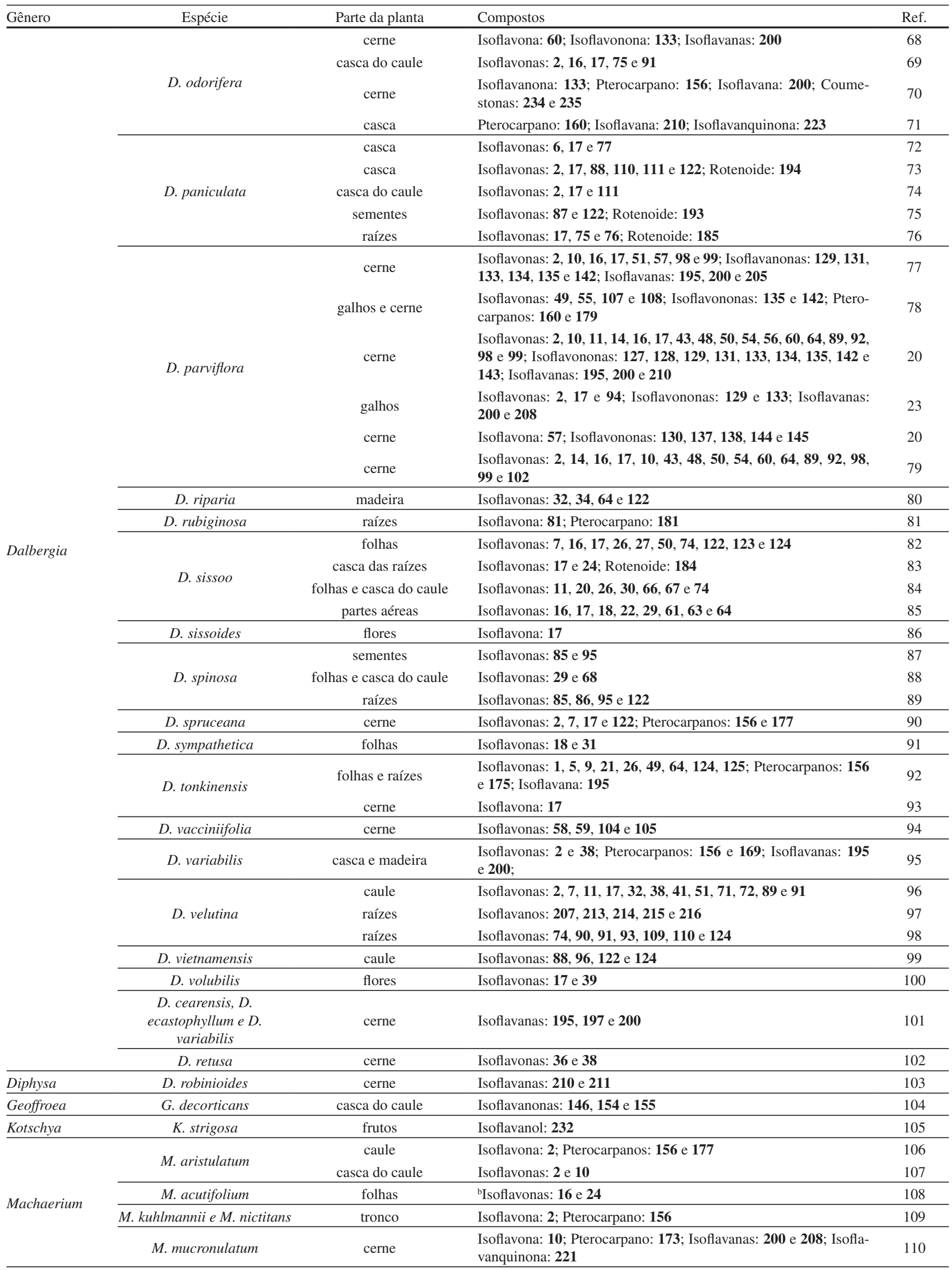


Tabela 1. Isoflavonoides de espécies da tribo Dalbergieae (cont.)

\begin{tabular}{|c|c|c|c|c|}
\hline Gênero & Espécie & Parte da planta & Compostos & Ref. \\
\hline \multirow{4}{*}{ Machaerium } & M. opacum & cerne & Isoflavanas: 200 e 208 & 111 \\
\hline & M. pedicellatum & cerne & Isoflavanas: 218 e 219 & 112 \\
\hline & M. vestitum & cerne & Isoflavona: 2; Pterocarpanos: 156 e 165; Isoflavanas: 195 e 200 & 113 \\
\hline & M. villosum & madeira & $\begin{array}{l}\text { Isoflavonas: 1, 3, } 9 \text { e 10; Isoflavanas: } 200 \text { e 208; Pterocarpanos: } \\
157 \text { e } \mathbf{1 7 3}\end{array}$ & 110 \\
\hline \multirow{3}{*}{ Ormocarpum } & \multirow{3}{*}{ O. kirkii } & casca do caule e raízes & Isoflavonas: 84 e 120 & 114 \\
\hline & & raízes & Isoflavanonas: 147, 148 e 149 & 115 \\
\hline & & raízes & Isoflavanona: 147 & 116 \\
\hline \multirow{6}{*}{ Platymiscium } & \multirow{4}{*}{ P. floribundum } & & Pterocarpano: 156 & 117 \\
\hline & & cerne & Pterocarpanos: 156, 157, 163165 e 170 & 118 \\
\hline & & tronco & Pterocarpanos: 156, 157, 163, 165, 170 & 119 \\
\hline & & tronco & Isoflavona: 100; Pterocarpanos: 156, 157, 163, 165 e 170 & 120 \\
\hline & P. gracile & serragem & Isoflavona: 10; Pterocarpano: 157 & 121 \\
\hline & P. yucatanum & tronco & Pterocarpanos: 156 e 157 & 122 \\
\hline \multirow{13}{*}{ Pterocarpus } & \multirow{2}{*}{ P. erinaceus } & casca dos galhos & Isoflavonas: 10; Isoflavonoide benzofurano: $\mathbf{2 4 0}$ & 123 \\
\hline & & casca dos galhos & Pterocarpano: 157 & 124 \\
\hline & \multirow{2}{*}{ P. indicus } & raízes & Isoflavonas: $\mathbf{2}, \mathbf{1 0}, \mathbf{1 7}, \mathbf{1 9}, 32$ e 38; Pterocarpano: 156 e 160 & 125 \\
\hline & & cerne & Isoflavona: 2; Pterocarpano: 159 & 126 \\
\hline & \multirow{4}{*}{ P. macrocarpus } & cerne & Isoflavanona: 150 & 127 \\
\hline & & planta & Isoflavona: $\mathbf{2 3}$ & 128 \\
\hline & & cerne & Isoflavonas: 8,40 e 62 & 129 \\
\hline & & cerne & Isoflavona: $\mathbf{7 3}$ & 130 \\
\hline & \multirow{3}{*}{ P. santalinus } & cerne & Isoflavona: $\mathbf{5 3}$ & 131 \\
\hline & & cerne & Isoflavona: 97 & 132 \\
\hline & & cerne & Isoflavona: $\mathbf{5 2}$ & 133 \\
\hline & \multirow[b]{2}{*}{ P. soyauxii } & cerne & Isoflavanas: 200, 201, 218 e 220; Isoflavanquinona: 222 & 134 \\
\hline & & cerne & $\begin{array}{l}\text { Isoflavonas: } 10,12,13,37,43,78,98,99,101,102,113,114 \text { e } \\
115 \text {; Pterocarpanos: } 157,159,160,161 \text { e } 167\end{array}$ & 135 \\
\hline \multirow{3}{*}{ Vatairea } & \multirow{3}{*}{ V. guianensis } & folhas & Isoflavonas: 11, 79 e 118 & 136 \\
\hline & & alburno & Isoflavona: 2; Isoflavonol: $\mathbf{2 3 3}$ & 137 \\
\hline & & folhas & Isoflavonas: 80, 82, 83 e 117 & 138 \\
\hline Zornia & Z. brasiliensis & partes aérias & Isoflavonas: 4 e 5; Pterocarpano: 156 & 139 \\
\hline
\end{tabular}

${ }^{\mathrm{a} C o m p o s t o s}$ identificados por CLAE-EM; ${ }^{\mathrm{b}}$ Compostos identificados por IT-ESI-MS.

um total de $125(52,1 \%)$. As isoflavonas ( $\mathbf{1}$ a $\mathbf{1 2 5})$ foram agrupadas com base em padrões simples de substituição no oxigênio, sendo 66 compostos com grupos metoxi e metilenodioxi, 12 com substituintes prenila e 42 derivados glicosilados. ${ }^{6}$

As isoflavonas têm 3-fenil-4H-1-benzopiran-4-ona (3-fenilcromona) como um sistema de anel principal ${ }^{13}$ e possuem em sua estrutura uma ligação dupla no carbono 2 do anel C (Tabela 2) e as que possuem substituição C-8 (hidroxi, metoxi ou derivados glicosidados) no anel A são relativamente incomuns, contudo, 19 compostos com esse padrão de substituição foram relatados (Tabela 2).

Um total de 35 isoflavonas da tribo Dalbergieae $(28,0 \%)$ são biologicamente ativas. As atividades biológicas mais frequentes em isoflavonas são estrogênica, citotóxica, antioxidante, antimicrobiana e antifúngica.

A atividade estrogênica foi relatada para 15 isoflavonas $(\mathbf{2}, \mathbf{1 1}, \mathbf{1 4}$, $16,17,48,50,54,57,60,64,89,92,98$ e 124) com ocorrência em 11 espécies, sendo a maioria encontrada na madeira de $D$. parviflora, o que indica o potencial estrogênico desta espécie.

Formononetina (2), genisteína (16), biochanina A (17), cajanina (57) e khrinona $\mathrm{C}(\mathbf{9 8})$ apresentaram ainda atividade antioxidante nos métodos Xantina/Xantina Oxidase (X/XO), capacidade de absorção do radical oxigênio (ORAC) e DPPH. ${ }^{141}$ Essa atividade também foi relatada para daidzeína (1), isoformononetina (3) e 3'-hidroxidaidzeína (9), calicosina (10), 3'-O-metilorobol (51) e khrinona B (99). ${ }^{13,123,141}$
Orobol (49), obtida de D. parviflora, apresentou atividade citotóxica frente às linhagens de células de carcionoma epidemoide de cavidade oral (KB), adenocarcinoide de mama (MCF-7) e câncer de pulmão de pequenas células (NCI-H187) ${ }^{78} \mathrm{~A}$ citotoxicidade frente às linhagens de células KB e NCI-H187 também foi exibida por 5,7,5'-trihidroxi-2',4'-dimetoxi-isoflavona (94), obtida de $D$. parviflo$r a{ }^{66,96}$ Enquanto 3'-metoxidaidzeína (11), obtida de $D$. odorifera e $D$. velutina, e pseudobaptigenina (7), isolada de D. velutina, foram ativas frente à linhagem de células $\mathrm{KB} .{ }^{23,96}$ 6,2'-Dimetoxi-7,4'-dihidroxiisoflavona (58), isolada de D. vacciniifolia e 5,7-dihidroxi-4'-metoxi-6,8-diprenilisoflavona (120), obtida de $O$. kirkii, demonstraram citotoxicidade frente a larvas de camarão e à linhagem de célula de leucemia (CCRF-CEM), respectivamente. ${ }^{94,114}$

A isoflavona biochanina A (17) apresentou atividade anti-inflamatória e antimicrobiana. ${ }^{90,97}$ Formononetina (2) e calicosina (10) demostraram potencial antimicrobiano, antiparasitário e antigiárdico contra Giardia intestinalis. ${ }^{13,48,107} \mathrm{O}$ composto 2 possui ainda atividade larvicida frente a Aedes aegypti, repelente contra zoósporo e inibidora de agregação plaquetária. ${ }^{62,64,67}$

A atividade antimalárica frente a Plasmodium falciparum é relatada para calicosina (10), genisteína (16) e 5,7,5'-trihidroxi$2^{\prime}, 4^{\prime}$-dimetoxi-isoflavona (94). ${ }^{23,32}$ Calicosina (10), obtida de $C$. sclerophyllum, apresentou também atividade leishmanicida. ${ }^{38}$ 5-Hidroxibowdichiona (107), obtida de D. candenatensis, e 
5,7,5'-trihidroxi-2', $4^{\prime}$-dimetoxiisoflavona (94) apresentaram atividade antimicrobiana. ${ }^{23,41}$ Enquanto calicosina (10) e 5,7,3'-trihidroxi-4'-metoxi-8-prenilisoflavona (119), isolada de V. guianensis, exibiram potencial antifúngico frente a cepas de Candida. ${ }^{121,136}$

Biochanina A 7-O- $\beta$-D-glicopiranosídeo (22), obtida de $A$. anthelmia e $D$. sissoo, biochanina A 7-O- $\beta$-D-apiofuranosil- $(1 \rightarrow 5)-$ $\beta$-D-apiofuranosil-( $1 \rightarrow 6)-\beta$-D-glicopiranosídeo $(27)$ e biochanina A 7-O- $\alpha$-L-ramnopiranosil- $(1 \rightarrow 6)-\beta$-D-glicopiranosídeo (28), isoladas de $A$. anthelmia, apresentaram atividade anti-helmíntica contra Aspiculuris tetraptera.$^{28} \mathrm{~A}$ atividade inibitória da ativação do antígeno do vírus Epstein Barr foi relatada para formononetina (2), genisteína (16), biochanina A (17), olibergina B (75) e olibergina A (91), obtidas de D. olivari. ${ }^{69}$

A partir do extrato metanólico da madeira de $D$. odorifera foi obtida uma fração que apresentou atividade anti-hipercolesterêmica e desta foram isoladas as isoflavonas formononetina (2) e 3'-metoxidaidzeína (11) ${ }^{65} \mathrm{~A}$ atividade inibitória de $\alpha$-glicosidase foi apresentada pela tectorigenina (64), obtida de D. odorifera e D. parviflora, pterosonina E (13), isolada de $D$. parviflora, e pelo composto $2 .^{22,61}$ Finalmente, a isoflavona 7-O- $\alpha$-L-ramnopiranosiloxi-4-metoxi-5hidroxi-isoflavona (23), obtida de $P$. marsupium, exibiu atividade reguladora de Glut-4 e PPAR. ${ }^{128}$

Tabela 2. Estruturas das isoflavonas de 1-125<smiles>[R3]c1cc(-c2coc3c([R])c([R])c([R])c([R])c3c2=O)cc([R])c1[R]</smiles><smiles>COc1cc2c(cc1-c1coc3cc(O)ccc3c1=O)OCO2</smiles>

35 cuneatina<smiles>[R3]C1=CC(=O)C(c2coc3cc(O)c([R])c([R])c3c2=O)=CC1=O</smiles>

48 browdichiona $\left(\mathrm{R}_{1}, \mathrm{R}_{2}=\mathrm{H} ; \mathrm{R}_{3}=\mathrm{OCH}_{3}\right)$ 1007 - hidroxi-6,4'-dimetoxi-isoflavoquinona $\left(\mathrm{R}_{1}=\mathrm{OCH}_{3} ; \mathrm{R}_{2}=\mathrm{H} ; \mathrm{R}_{3}=\mathrm{CH}_{3}\right)$

107 5-hidrobrowdichiona $\left(\mathrm{R}_{1}=\mathrm{H} ; \mathrm{R}_{2}=\mathrm{OH} ; \mathrm{R}_{3}=\mathrm{OCH}_{3}\right)$

\begin{tabular}{|c|c|c|c|c|c|c|c|c|c|}
\hline $\mathrm{N}^{\mathrm{o}}$ & $\mathrm{R}_{8}$ & $\mathrm{R}_{7}$ & $\mathrm{R}_{6}$ & $\mathrm{R}_{5}$ & $\mathrm{R}_{2}$, & $\mathrm{R}_{3}$, & $\mathrm{R}_{4}$, & $\mathrm{R}_{5}$, & Nome \\
\hline 1 & $\mathrm{H}$ & $\mathrm{OH}$ & $\mathrm{H}$ & $\mathrm{H}$ & $\mathrm{H}$ & $\mathrm{H}$ & $\mathrm{OH}$ & $\mathrm{H}$ & daidzeína \\
\hline 2 & $\mathrm{H}$ & $\mathrm{OH}$ & $\mathrm{H}$ & $\mathrm{H}$ & $\mathrm{H}$ & $\mathrm{H}$ & $\mathrm{OCH}_{3}$ & $\mathrm{H}$ & formononetina \\
\hline 3 & $\mathrm{H}$ & $\mathrm{OCH}_{3}$ & $\mathrm{H}$ & $\mathrm{H}$ & $\mathrm{H}$ & $\mathrm{H}$ & $\mathrm{OH}$ & $\mathrm{H}$ & isoformononetina \\
\hline 4 & $\mathrm{H}$ & $\mathrm{OCH}_{3}$ & $\mathrm{H}$ & $\mathrm{H}$ & $\mathrm{H}$ & $\mathrm{H}$ & $\mathrm{OCH}_{3}$ & $\mathrm{H}$ & 7,4'-dimetoxi-isoflavona \\
\hline 5 & $\mathrm{H}$ & $O-\beta$-D-glc & $\mathrm{H}$ & $\mathrm{H}$ & $\mathrm{H}$ & $\mathrm{H}$ & $\mathrm{OCH}_{3}$ & $\mathrm{H}$ & ononina \\
\hline 6 & $\mathrm{H}$ & $\begin{array}{c}O-\beta \text {-L-rha- }(1 \rightarrow 6)- \\
\beta-\mathrm{D}-\mathrm{glc}\end{array}$ & $\mathrm{H}$ & $\mathrm{H}$ & $\mathrm{H}$ & $\mathrm{H}$ & $\mathrm{OCH}_{3}$ & $\mathrm{H}$ & formononetina 7-rutinosídeo \\
\hline 7 & $\mathrm{H}$ & $\mathrm{OH}$ & $\mathrm{H}$ & $\mathrm{H}$ & $\mathrm{H}$ & $\mathrm{O}-\mathrm{CH}_{2}-\mathrm{O}$ & & $\mathrm{H}$ & pseudobaptigenina \\
\hline 8 & $\mathrm{H}$ & $O-\alpha$-L-rha & $\mathrm{OCH}_{3}$ & $\mathrm{OH}$ & $\mathrm{H}$ & $\mathrm{H}$ & $\mathrm{H}$ & $\mathrm{H}$ & $\begin{array}{c}\text { 5,7-dihidroxi-6-metoxi-isoflavona } \\
\text { 7-rha }\end{array}$ \\
\hline 9 & $\mathrm{H}$ & $\mathrm{OH}$ & $\mathrm{H}$ & $\mathrm{H}$ & $\mathrm{H}$ & $\mathrm{OH}$ & $\mathrm{OH}$ & $\mathrm{H}$ & 3'-hidroxidaidzeína \\
\hline 10 & $\mathrm{H}$ & $\mathrm{OH}$ & $\mathrm{H}$ & $\mathrm{H}$ & $\mathrm{H}$ & $\mathrm{OH}$ & $\mathrm{OCH}_{3}$ & $\mathrm{H}$ & calicosina \\
\hline 11 & $\mathrm{H}$ & $\mathrm{OH}$ & $\mathrm{H}$ & $\mathrm{H}$ & $\mathrm{H}$ & $\mathrm{OCH}_{3}$ & $\mathrm{OH}$ & $\mathrm{H}$ & 3'-metoxidaidzeína \\
\hline 12 & $\mathrm{H}$ & $\mathrm{OCH}_{3}$ & $\mathrm{H}$ & $\mathrm{H}$ & $\mathrm{H}$ & $\mathrm{OCH}_{3}$ & $\mathrm{OH}$ & $\mathrm{H}$ & saianedina \\
\hline 13 & $\mathrm{H}$ & $\mathrm{OH}$ & $\mathrm{H}$ & $\mathrm{H}$ & $\mathrm{H}$ & $\mathrm{OH}$ & $\mathrm{H}$ & $\mathrm{OCH}_{3}$ & pterosonina $\mathrm{E}$ \\
\hline 14 & $\mathrm{H}$ & $\mathrm{OH}$ & $\mathrm{H}$ & $\mathrm{H}$ & $\mathrm{OCH}_{3}$ & $\mathrm{H}$ & $\mathrm{OCH}_{3}$ & $\mathrm{H}$ & 2'-metoxiformononetina \\
\hline 15 & $\mathrm{H}$ & $\mathrm{OH}$ & $\mathrm{H}$ & $\mathrm{H}$ & $\mathrm{OH}$ & $\mathrm{H}$ & $\mathrm{OCH}_{3}$ & $\mathrm{H}$ & 2'-hidroxiformononetina \\
\hline 16 & $\mathrm{H}$ & $\mathrm{OH}$ & $\mathrm{H}$ & $\mathrm{OH}$ & $\mathrm{H}$ & $\mathrm{H}$ & $\mathrm{OH}$ & $\mathrm{H}$ & genisteína \\
\hline 17 & $\mathrm{H}$ & $\mathrm{OH}$ & $\mathrm{H}$ & $\mathrm{OH}$ & $\mathrm{H}$ & $\mathrm{H}$ & $\mathrm{OCH}_{3}$ & $\mathrm{H}$ & biochanina $\mathrm{A}$ \\
\hline 18 & $\mathrm{H}$ & $\mathrm{OCH}_{3}$ & $\mathrm{H}$ & $\mathrm{OH}$ & $\mathrm{H}$ & $\mathrm{H}$ & $\mathrm{OH}$ & $\mathrm{H}$ & prunetina \\
\hline 19 & $\mathrm{H}$ & $\mathrm{OH}$ & $\mathrm{H}$ & $\mathrm{OCH}_{3}$ & $\mathrm{H}$ & $\mathrm{H}$ & $\mathrm{OCH}_{3}$ & $\mathrm{H}$ & 5-O-metilbiochanina A \\
\hline 20 & $\mathrm{H}$ & $\mathrm{OCH}_{3}$ & $\mathrm{H}$ & $\mathrm{OH}$ & $\mathrm{H}$ & $\mathrm{H}$ & $\begin{array}{c}O-\beta \text {-D-apio- } \\
(1 \rightarrow 6)-\beta \text {-D-glc }\end{array}$ & $\mathrm{H}$ & coromandelina; \\
\hline 21 & $\mathrm{H}$ & $\begin{array}{c}O \text { - } \beta \text {-D-apio- }(1 \rightarrow 6)- \\
\beta \text {-D-glc }\end{array}$ & $\mathrm{H}$ & $\mathrm{OH}$ & $\mathrm{H}$ & $\mathrm{H}$ & $\mathrm{OH}$ & $\mathrm{H}$ & ambocina \\
\hline 22 & $\mathrm{H}$ & $O-\beta-\mathrm{D}-\mathrm{glc}$ & $\mathrm{H}$ & $\mathrm{OH}$ & $\mathrm{H}$ & $\mathrm{H}$ & $\mathrm{OCH}_{3}$ & $\mathrm{H}$ & biochanina A $7-O-\beta-\mathrm{D}-\mathrm{glc}$ \\
\hline 23 & $\mathrm{H}$ & $O$ - $\alpha$-L-rha & $\mathrm{H}$ & $\mathrm{OH}$ & $\mathrm{H}$ & $\mathrm{H}$ & $\mathrm{OCH}_{3}$ & $\mathrm{H}$ & $\begin{array}{l}\text { 7-O- } \alpha \text {-L-rha-4-metoxi-5-hidroxi- } \\
\text { isoflavona }\end{array}$ \\
\hline 24 & $\mathrm{H}$ & $\gamma, \gamma$-dimetilalliloxi & $\mathrm{H}$ & $\mathrm{OH}$ & $\mathrm{H}$ & $\mathrm{H}$ & $\mathrm{OCH}_{3}$ & $\mathrm{H}$ & $\begin{array}{l}\text { 7- } \gamma, \gamma \text {-dimetilalliloxi-5-hidroxi-4'- } \\
\text { metoxi-isoflavona }\end{array}$ \\
\hline 25 & $\mathrm{H}$ & $O$-xil $(1 \rightarrow 6)$-glc & $\mathrm{H}$ & $\mathrm{OH}$ & $\mathrm{H}$ & $\mathrm{H}$ & $\mathrm{OCH}_{3}$ & $\mathrm{H}$ & $\begin{array}{c}\text { biochanina A 7-O- } \beta \text {-D-xil- }(1 \rightarrow 6)- \\
\beta-\mathrm{D}-\mathrm{glc}\end{array}$ \\
\hline 26 & $\mathrm{H}$ & $\begin{array}{c}O \text { - } \beta \text {-D-apio- }(1 \rightarrow 6)- \\
\beta \text {-D-glc }\end{array}$ & $\mathrm{H}$ & $\mathrm{OH}$ & $\mathrm{H}$ & $\mathrm{H}$ & $\mathrm{OCH}_{3}$ & $\mathrm{H}$ & lanceolarina \\
\hline 27 & $\mathrm{H}$ & $\begin{array}{c}O-\beta \text {-D-apio- }(1 \rightarrow 5)- \\
\beta \text {-D-apio- }(1 \rightarrow 6)-\beta- \\
\text { D-glc } \\
\end{array}$ & $\mathrm{H}$ & $\mathrm{OH}$ & $\mathrm{H}$ & $\mathrm{H}$ & $\mathrm{OCH}_{3}$ & $\mathrm{H}$ & $\begin{array}{c}\text { biochanina A 7-O- } \beta \text {-D-apio- } \\
(1 \rightarrow 5)-\beta-\mathrm{D} \text {-apio- }(1 \rightarrow 6)-\beta-\mathrm{D} \text {-glc }\end{array}$ \\
\hline
\end{tabular}


Tabela 2. Estruturas das isoflavonas de 1-125 (cont.)

\begin{tabular}{|c|c|c|c|c|c|c|c|c|c|}
\hline $\mathrm{N}^{\mathrm{o}}$ & $\mathrm{R}_{8}$ & $\mathrm{R}_{7}$ & $\mathrm{R}_{6}$ & $\mathrm{R}_{5}$ & $\mathrm{R}_{2}$ & $\mathrm{R}_{3}$ & $\mathrm{R}_{4}$ & $\mathrm{R}_{5}$ & Nome \\
\hline 28 & $\mathrm{H}$ & $\begin{array}{c}O-\alpha-\mathrm{L}-\mathrm{rha}-(1 \rightarrow 6)- \\
\beta-\mathrm{D}-\mathrm{glc}\end{array}$ & $\mathrm{H}$ & $\mathrm{OH}$ & $\mathrm{H}$ & $\mathrm{H}$ & $\mathrm{OCH}_{3}$ & $\mathrm{H}$ & $\begin{array}{l}\text { biochanina A } 7-O-\alpha-\text { L-rha- } \\
\qquad(1 \rightarrow 6)-\beta-\mathrm{D}-\mathrm{glc}\end{array}$ \\
\hline 29 & $\mathrm{H}$ & $\mathrm{OCH}_{3}$ & $\mathrm{H}$ & $\mathrm{OH}$ & $\mathrm{H}$ & $\mathrm{H}$ & $O-\beta-\mathrm{D}-\mathrm{gal}$ & $\mathrm{H}$ & prunetina 4'- $O-\beta-\mathrm{D}-\mathrm{gal}$ \\
\hline 30 & $\mathrm{H}$ & $\mathrm{OCH}_{3}$ & $\mathrm{H}$ & $\mathrm{OH}$ & $\mathrm{H}$ & $\mathrm{H}$ & $O-\beta-\mathrm{D}-\mathrm{glc}$ & $\mathrm{H}$ & prunetina 4'-O- $\beta-\mathrm{D}-\mathrm{glc}$ \\
\hline 31 & $\mathrm{H}$ & $\mathrm{OCH}_{3}$ & $\mathrm{H}$ & $\mathrm{OH}$ & $\mathrm{H}$ & $\mathrm{H}$ & $O-\beta$-D-gen & $\mathrm{H}$ & dalsimpatetina \\
\hline 32 & $\mathrm{H}$ & $\mathrm{OH}$ & $\mathrm{OCH}_{3}$ & $\mathrm{H}$ & $\mathrm{H}$ & $\mathrm{H}$ & $\mathrm{OCH}_{3}$ & $\mathrm{H}$ & afrormosina \\
\hline 33 & $\mathrm{H}$ & $\mathrm{OH}$ & $\mathrm{OCH}_{3}$ & $\mathrm{H}$ & $\mathrm{H}$ & $\mathrm{H}$ & $\mathrm{OH}$ & $\mathrm{H}$ & gliciteina \\
\hline 34 & $\mathrm{H}$ & $\mathrm{OH}$ & $\mathrm{OCH}_{3}$ & $\mathrm{H}$ & $\mathrm{H}$ & $\mathrm{O}-\mathrm{CH}_{2}-\mathrm{O}$ & & $\mathrm{H}$ & fujikinetina \\
\hline 36 & $\mathrm{OH}$ & $\mathrm{OH}$ & $\mathrm{H}$ & $\mathrm{H}$ & $\mathrm{H}$ & $\mathrm{H}$ & $\mathrm{OCH}_{3}$ & $\mathrm{H}$ & retusina \\
\hline 37 & $\mathrm{OH}$ & $\mathrm{OCH}_{3}$ & $\mathrm{H}$ & $\mathrm{H}$ & $\mathrm{H}$ & $\mathrm{H}$ & $\mathrm{OCH}_{3}$ & $\mathrm{H}$ & 8-hidroxi-4',7-dimetoxi-isoflavona \\
\hline 38 & $\mathrm{OCH}_{3}$ & $\mathrm{OH}$ & $\mathrm{H}$ & $\mathrm{H}$ & $\mathrm{H}$ & $\mathrm{H}$ & $\mathrm{OCH}_{3}$ & $\mathrm{H}$ & 8-O-metilretusina \\
\hline 39 & rha & $\mathrm{OCH}_{3}$ & $\mathrm{H}$ & $\mathrm{H}$ & $\mathrm{H}$ & $\mathrm{H}$ & $\mathrm{OCH}_{3}$ & $\mathrm{H}$ & volubilina \\
\hline 40 & $\mathrm{OH}$ & $O-\beta-\mathrm{D}-\mathrm{glc}$ & $\mathrm{H}$ & $\mathrm{H}$ & $\mathrm{H}$ & $\mathrm{H}$ & $\mathrm{OCH}_{3}$ & $\mathrm{H}$ & retusina $7-O-\beta-\mathrm{D}-\mathrm{glc}$ \\
\hline 41 & $\mathrm{H}$ & $\mathrm{OH}$ & $\mathrm{H}$ & $\mathrm{OH}$ & $\mathrm{H}$ & $\mathrm{O}-\mathrm{CH}_{2}-\mathrm{O}$ & & $\mathrm{H}$ & 3',4'-metilenodioxi orobol \\
\hline 42 & $\mathrm{H}$ & $\mathrm{OH}$ & $\mathrm{H}$ & $\mathrm{H}$ & $\mathrm{OCH}_{3}$ & $\mathrm{H}$ & $\mathrm{OH}$ & $\mathrm{OH}$ & $\begin{array}{l}4^{\prime}, 5^{\prime}, 7 \text {-trihidroxi-2'-metoxi- } \\
\text { isoflavona }\end{array}$ \\
\hline 43 & $\mathrm{H}$ & $\mathrm{OH}$ & $\mathrm{H}$ & $\mathrm{H}$ & $\mathrm{OH}$ & $\mathrm{H}$ & $\mathrm{OCH}_{3}$ & $\mathrm{OH}$ & khrinona A \\
\hline 44 & $\mathrm{H}$ & $\mathrm{OH}$ & $\mathrm{H}$ & $\mathrm{H}$ & $\mathrm{OH}$ & $\mathrm{H}$ & $\mathrm{OCH}_{3}$ & $\mathrm{OCH}_{3}$ & $\begin{array}{l}\text { 2',7-dihidroxi-4',5'-dimetoxi- } \\
\text { isoflavona }\end{array}$ \\
\hline 45 & $\mathrm{H}$ & $\mathrm{OH}$ & $\mathrm{H}$ & $\mathrm{H}$ & $\mathrm{OCH}_{3}$ & $\mathrm{H}$ & $\mathrm{OCH}_{3}$ & $\mathrm{OCH}_{3}$ & $\begin{array}{l}\text { 7-hidroxi-2',4',5' -trimetoxi- } \\
\text { isoflavona }\end{array}$ \\
\hline 46 & $\mathrm{H}$ & $O-\beta-\mathrm{D}-\mathrm{glc}$ & $\mathrm{H}$ & $\mathrm{H}$ & $\mathrm{OCH}_{3}$ & $\mathrm{H}$ & $\mathrm{OCH}_{3}$ & $\mathrm{OCH}_{3}$ & $\begin{array}{c}\text { 7- } \beta \text {-D-glc-2',4',5'-trimetoxi- } \\
\text { isoflavona }\end{array}$ \\
\hline 47 & $\mathrm{H}$ & $O-\beta-\mathrm{D}-\mathrm{glc}$ & $\mathrm{H}$ & $\mathrm{H}$ & $\mathrm{OH}$ & $\mathrm{H}$ & $\mathrm{OCH}_{3}$ & $\mathrm{OCH}_{3}$ & daidzeína 7-O- $\beta$-D-glc \\
\hline 49 & $\mathrm{H}$ & $\mathrm{OH}$ & $\mathrm{H}$ & $\mathrm{OH}$ & $\mathrm{H}$ & $\mathrm{OH}$ & $\mathrm{OH}$ & $\mathrm{H}$ & orobol \\
\hline 50 & $\mathrm{H}$ & $\mathrm{OH}$ & $\mathrm{H}$ & $\mathrm{OH}$ & $\mathrm{H}$ & $\mathrm{OH}$ & $\mathrm{OCH}_{3}$ & $\mathrm{H}$ & pratenseína \\
\hline 51 & $\mathrm{H}$ & $\mathrm{OH}$ & $\mathrm{H}$ & $\mathrm{OH}$ & $\mathrm{H}$ & $\mathrm{OCH}_{3}$ & $\mathrm{OH}$ & $\mathrm{H}$ & 3'-O-metilorobol \\
\hline 52 & $\mathrm{H}$ & $\mathrm{OCH}_{3}$ & $\mathrm{H}$ & $\mathrm{OH}$ & $\mathrm{H}$ & $O-\beta-\mathrm{D}-\mathrm{glc}$ & $\mathrm{OH}$ & $\mathrm{H}$ & $\begin{array}{l}\text { 4',5-dihidroxi-7- } O \text { - } \\
\text { metilisoflavona 3'- } O-\beta \text {-D-glc }\end{array}$ \\
\hline 53 & $\mathrm{H}$ & $\mathrm{OCH}_{3}$ & $\mathrm{H}$ & $\mathrm{OH}$ & $\mathrm{H}$ & $\begin{array}{c}O-\beta-\mathrm{D}- \\
(3 "-E \text {-cinamoil })- \\
\text { glc }\end{array}$ & $\mathrm{OH}$ & $\mathrm{H}$ & $\begin{array}{c}\text { 4',5-dihidroxi-7-O- } \\
\text { metilisoflavona 3'-O- } \beta \text {-D- } \\
\text { (3'”-E-cinamoil)-glc }\end{array}$ \\
\hline 54 & $\mathrm{H}$ & $\mathrm{OH}$ & $\mathrm{H}$ & $\mathrm{OH}$ & $\mathrm{OCH}_{3}$ & $\mathrm{H}$ & $\mathrm{OH}$ & $\mathrm{H}$ & teralina \\
\hline 55 & $\mathrm{H}$ & $\mathrm{OH}$ & $\mathrm{H}$ & $\mathrm{OH}$ & $\mathrm{OH}$ & $\mathrm{H}$ & $\mathrm{OCH}_{3}$ & $\mathrm{H}$ & 2'-hidroxibiochanina A \\
\hline 56 & $\mathrm{H}$ & $\mathrm{OH}$ & $\mathrm{H}$ & $\mathrm{OH}$ & $\mathrm{OCH}_{3}$ & $\mathrm{H}$ & $\mathrm{OCH}_{3}$ & $\mathrm{H}$ & 2'-metoxibiochanina A \\
\hline 57 & $\mathrm{H}$ & $\mathrm{OCH}_{3}$ & $\mathrm{H}$ & $\mathrm{OH}$ & $\mathrm{OH}$ & $\mathrm{H}$ & $\mathrm{OH}$ & $\mathrm{H}$ & cajanina \\
\hline 58 & $\mathrm{H}$ & $\mathrm{OH}$ & $\mathrm{OCH}_{3}$ & $\mathrm{H}$ & $\mathrm{OCH}_{3}$ & $\mathrm{H}$ & $\mathrm{OH}$ & $\mathrm{H}$ & $\begin{array}{l}6,2 \text { '-dimetoxi-7,4'-dihidroxi- } \\
\text { isoflavona }\end{array}$ \\
\hline 59 & $\mathrm{H}$ & $\mathrm{OH}$ & $\mathrm{OCH}_{3}$ & $\mathrm{H}$ & $\mathrm{OCH}_{3}$ & $\mathrm{H}$ & $\mathrm{OCH}_{3}$ & $\mathrm{H}$ & $\begin{array}{l}6,2 \text { ',4'-trimetoxi-7-hidroxi- } \\
\text { isoflavona }\end{array}$ \\
\hline 60 & $\mathrm{H}$ & $\mathrm{OH}$ & $\mathrm{H}$ & $\mathrm{H}$ & $\mathrm{OCH}_{3}$ & $\mathrm{OH}$ & $\mathrm{OCH}_{3}$ & $\mathrm{H}$ & khrinona $\mathrm{E}$ \\
\hline 61 & $\mathrm{H}$ & $\mathrm{OH}$ & $\mathrm{OCH}_{3}$ & $\mathrm{H}$ & $\mathrm{H}$ & $\mathrm{H}$ & $\mathrm{OCH}_{3}$ & $\mathrm{H}$ & irisolidona \\
\hline 62 & $\mathrm{H}$ & $O-\alpha-\mathrm{L}-\mathrm{rha}$ & $\mathrm{OCH}_{3}$ & $\mathrm{OH}$ & $\mathrm{H}$ & $\mathrm{H}$ & $\mathrm{OCH}_{3}$ & $\mathrm{H}$ & irisolidona $7-O-\alpha-\mathrm{L}-\mathrm{rha}$ \\
\hline 63 & $\mathrm{H}$ & $\mathrm{OCH}_{3}$ & $\mathrm{OH}$ & $\mathrm{OCH}_{3}$ & $\mathrm{H}$ & $\mathrm{H}$ & $\mathrm{OH}$ & $\mathrm{H}$ & muningina \\
\hline 64 & $\mathrm{H}$ & $\mathrm{OH}$ & $\mathrm{OCH}_{3}$ & $\mathrm{OH}$ & $\mathrm{H}$ & $\mathrm{H}$ & $\mathrm{OH}$ & $\mathrm{H}$ & tectorigenina \\
\hline 65 & $\mathrm{H}$ & $\mathrm{OCH}_{3}$ & $\mathrm{OCH}_{3}$ & $\mathrm{OH}$ & $\mathrm{H}$ & $\mathrm{H}$ & $\mathrm{OH}$ & $\mathrm{H}$ & 7-O-metiltectorigenina \\
\hline 66 & $\mathrm{H}$ & $\begin{array}{c}O-\beta \text {-D-apio- }(1 \rightarrow 6)- \\
\beta-\mathrm{D}-\mathrm{glc}\end{array}$ & $\mathrm{OCH}_{3}$ & $\mathrm{OH}$ & $\mathrm{H}$ & $\mathrm{H}$ & $\mathrm{OH}$ & $\mathrm{H}$ & $\begin{array}{l}\text { tectorigenina } 7-O-\beta-\mathrm{D} \text {-apio- } \\
\qquad(1 \rightarrow 6)-\beta-\mathrm{D}-\mathrm{glc}\end{array}$ \\
\hline 67 & $\mathrm{H}$ & $\mathrm{OCH}_{3}$ & $\mathrm{OCH}_{3}$ & $\mathrm{OH}$ & $\mathrm{H}$ & $\mathrm{H}$ & $\begin{array}{c}O-6-O-\beta-\mathrm{D}-\text { apio- } \\
\beta-\mathrm{D}-\mathrm{glc}\end{array}$ & $\mathrm{H}$ & $\begin{array}{l}\text { 5-hidroxi-6,7-dimetoxi-4'-O-6-O- } \\
\text { } \text {-D-apio- } \beta \text {-D-glc isoflavona }\end{array}$ \\
\hline 68 & $\mathrm{H}$ & $\mathrm{OCH}_{3}$ & $\mathrm{OCH}_{3}$ & $\mathrm{OH}$ & $\mathrm{H}$ & $\mathrm{H}$ & $O-\beta-\mathrm{D}-\mathrm{gal}$ & $\mathrm{H}$ & $\begin{array}{l}\text { 7-metiltectorigenina 4'-O- } \beta \text {-D- } \\
\text { galactosídeo }\end{array}$ \\
\hline 69 & $\mathrm{H}$ & $\mathrm{OH}$ & $\mathrm{OCH}_{3}$ & $\mathrm{H}$ & $\mathrm{H}$ & $\mathrm{OH}$ & $\mathrm{OCH}_{3}$ & $\mathrm{H}$ & odoratina \\
\hline 70 & $\mathrm{H}$ & $\mathrm{OH}$ & $\mathrm{H}$ & $\mathrm{H}$ & $\mathrm{OH}$ & $\mathrm{OH}$ & $\mathrm{OCH}_{3}$ & $\mathrm{H}$ & koparina \\
\hline 71 & $\mathrm{H}$ & $\mathrm{OH}$ & $\mathrm{H}$ & $\mathrm{H}$ & $\mathrm{H}$ & $\mathrm{OCH}_{3}$ & $\mathrm{OH}$ & $\mathrm{OCH}_{3}$ & veluisoflavona A \\
\hline 72 & $\mathrm{OCH}_{3}$ & $\mathrm{OH}$ & $\mathrm{H}$ & $\mathrm{H}$ & $\mathrm{H}$ & $\mathrm{H}$ & $\mathrm{OH}$ & $\mathrm{OCH}_{3}$ & veluisoflavona B \\
\hline 73 & $\mathrm{CH}_{3}$ & $O$ - $\alpha$-L-rha & $\mathrm{H}$ & $\mathrm{OCH}_{3}$ & $\mathrm{H}$ & $\mathrm{H}$ & $\mathrm{OCH}_{3}$ & $\mathrm{H}$ & $\begin{array}{c}\text { 5,4'-dimetoxi-8-metilisoflavona } \\
\text { 7-O- } \alpha \text {-L-rha }\end{array}$ \\
\hline
\end{tabular}


Tabela 2. Estruturas das isoflavonas de 1-125 (cont.)

\begin{tabular}{|c|c|c|c|c|c|c|c|c|c|}
\hline $\mathrm{N}^{\circ}$ & $\mathrm{R}_{8}$ & $\mathrm{R}_{7}$ & $\mathrm{R}_{6}$ & $\mathrm{R}_{5}$ & $\mathrm{R}_{2}$, & $\mathrm{R}_{3}$, & $\mathrm{R}_{4}$, & $\mathrm{R}_{5}$, & Nome \\
\hline 74 & $\beta$-D-glc & $\mathrm{OH}$ & $\mathrm{H}$ & $\mathrm{OH}$ & $\mathrm{H}$ & $\mathrm{H}$ & $\mathrm{OH}$ & $\mathrm{H}$ & genisteína-8-C- $\beta$-D-glc \\
\hline 75 & geranila & $\mathrm{OH}$ & $\mathrm{H}$ & $\mathrm{OH}$ & $\mathrm{H}$ & $\mathrm{H}$ & $\mathrm{OCH}_{3}$ & $\mathrm{H}$ & olibergina B \\
\hline 76 & geranila & $\mathrm{OCH}_{3}$ & $\mathrm{H}$ & $\mathrm{OH}$ & $\mathrm{H}$ & $\mathrm{H}$ & $\mathrm{OCH}_{3}$ & $\mathrm{H}$ & $\begin{array}{c}\text { 8-C-geranil-7- } O \text {-metilbiochanina } \\
\mathrm{A}\end{array}$ \\
\hline 77 & glc & $\mathrm{OCH}_{3}$ & $\mathrm{H}$ & $\mathrm{OH}$ & $\mathrm{H}$ & $\mathrm{H}$ & $\mathrm{OH}$ & $\mathrm{H}$ & prunetina $8-C$-glc \\
\hline 78 & $\mathrm{OH}$ & $\mathrm{OCH}_{3}$ & $\mathrm{H}$ & $\mathrm{H}$ & $\mathrm{H}$ & $\mathrm{OH}$ & $\mathrm{H}$ & $\mathrm{OCH}_{3}$ & pterosonina $\mathrm{D}$ \\
\hline 79 & prenila & $\mathrm{OH}$ & $\mathrm{H}$ & $\mathrm{OH}$ & $\mathrm{H}$ & $\mathrm{H}$ & $\mathrm{OH}$ & $\mathrm{H}$ & lupiwighteona \\
\hline 80 & prenila & $\mathrm{OH}$ & $\mathrm{H}$ & $\mathrm{OH}$ & $\mathrm{H}$ & $\mathrm{O}-\mathrm{CH}_{2}-\mathrm{O}$ & & $\mathrm{H}$ & $\begin{array}{c}\text { 5,7-dihidroxi-3',4'-metilenodioxi- } \\
\text { 8-prenilisoflavona }\end{array}$ \\
\hline 81 & $\mathrm{H}$ & $\mathrm{OCH}_{3}$ & $\mathrm{OCH}_{3}$ & $\mathrm{OH}$ & $\mathrm{H}$ & $\mathrm{O}-\mathrm{CH}_{2}-\mathrm{O}$ & & $\mathrm{H}$ & $\begin{array}{l}\text { 5-didroxi-6,7-dimetoxi-3',4'- } \\
\text { metilodioxi-isoflavona }\end{array}$ \\
\hline 82 & 2",2"-dimetil & Ipirano-(5",6":8,7) & $\mathrm{H}$ & $\mathrm{OH}$ & $\mathrm{H}$ & $\mathrm{H}$ & $\mathrm{OCH}_{3}$ & $\mathrm{H}$ & $\begin{array}{c}\text { 5,3'-dihidroxi-4'-metoxi-2",2"- } \\
\text { dimetilpirano-(5",6":8,7)- } \\
\text { isoflavona }\end{array}$ \\
\hline 83 & 2",2"-dimetil & Ipirano-(5",6":8,7) & $\mathrm{H}$ & $\mathrm{OH}$ & $\mathrm{H}$ & $\mathrm{H}$ & $\mathrm{OH}$ & $\mathrm{H}$ & derrona \\
\hline 84 & $2^{\prime}, 2^{\prime}$-dimetil & pirano- $(5 ", 6 ": 8,7)$ & prenila & $\mathrm{OH}$ & $\mathrm{H}$ & $\mathrm{H}$ & $\mathrm{OH}$ & $\mathrm{H}$ & osajina \\
\hline 85 & $\mathrm{H}$ & $\mathrm{OH}$ & $\mathrm{OCH}_{3}$ & $\mathrm{OH}$ & $\mathrm{H}$ & $\mathrm{O}-\mathrm{CH}_{2}-\mathrm{O}$ & & $\mathrm{H}$ & dalspinina \\
\hline 86 & $\mathrm{H}$ & $\begin{array}{l}O \text { - } \beta \text {-D-apio- } \\
(1 \rightarrow 6) \text {-glc }\end{array}$ & $\mathrm{OCH}_{3}$ & $\mathrm{OH}$ & $\mathrm{H}$ & $\mathrm{O}-\mathrm{CH}_{2}-\mathrm{O}$ & & $\mathrm{H}$ & $\begin{array}{c}\text { dalspinina-7- } O-\beta \text {-D-apio- }(1 \rightarrow 6)- \\
\text { glc }\end{array}$ \\
\hline 87 & $\mathrm{H}$ & $\mathrm{OH}$ & $\mathrm{OCH}_{3}$ & $\mathrm{H}$ & $\mathrm{OCH}_{3}$ & $\mathrm{O}-\mathrm{CH}_{2}-\mathrm{O}$ & & & dalpatiena \\
\hline 88 & $\mathrm{H}$ & $O-\beta-\mathrm{D}-\mathrm{glc}$ & $\mathrm{OCH}_{3}$ & $\mathrm{H}$ & $\mathrm{OCH}_{3}$ & $\mathrm{H}$ & $\mathrm{O}-\mathrm{CH}_{2}-\mathrm{O}$ & & dalpatina \\
\hline 89 & $\mathrm{H}$ & $\mathrm{OH}$ & $\mathrm{H}$ & $\mathrm{OH}$ & $\mathrm{OCH}_{3}$ & $\mathrm{H}$ & $\mathrm{O}-\mathrm{CH}_{2}-\mathrm{O}$ & & khrinona D \\
\hline 90 & $\mathrm{H}$ & $O-\beta-\mathrm{D}-\mathrm{glc}$ & $\mathrm{H}$ & $\mathrm{OH}$ & $\mathrm{OCH}_{3}$ & $\mathrm{H}$ & $\mathrm{OH}$ & $\mathrm{OCH}_{3}$ & dalvelutinosídeo \\
\hline 91 & $\mathrm{H}$ & $\mathrm{OH}$ & $\mathrm{H}$ & $\mathrm{OH}$ & $\mathrm{OCH}_{3}$ & $\mathrm{H}$ & $\mathrm{OH}$ & $\mathrm{OCH}_{3}$ & olibergina A \\
\hline 92 & $\mathrm{H}$ & $\mathrm{OH}$ & $\mathrm{H}$ & $\mathrm{OH}$ & $\mathrm{OCH}_{3}$ & $\mathrm{H}$ & $\mathrm{OCH}_{3}$ & $\mathrm{OCH}_{3}$ & 7-demetilrobustigenina \\
\hline 93 & $\mathrm{H}$ & $\begin{array}{c}O-\beta \text {-D-apio- } \\
(1 ", \rightarrow 6 ")-O-\beta-\mathrm{D}-\mathrm{glc}\end{array}$ & $\mathrm{H}$ & $\mathrm{OH}$ & $\mathrm{OCH}_{3}$ & $\mathrm{H}$ & $\mathrm{OH}$ & $\mathrm{OCH}_{3}$ & $\begin{array}{c}\text { 2',5'-dimetoxi-genisteína-7- } O-\beta \text { - } \\
\text { D-apio-(1'" } \rightarrow 6 \text { ")-O- } \beta \text {-D-glc }\end{array}$ \\
\hline 94 & $\mathrm{H}$ & $\mathrm{OH}$ & $\mathrm{H}$ & $\mathrm{OH}$ & $\mathrm{OCH}_{3}$ & $\mathrm{H}$ & $\mathrm{OCH}_{3}$ & $\mathrm{OH}$ & $\begin{array}{l}5,7,5^{\prime} \text {-trihidroxi-2',4'-dimetoxi- } \\
\text { isoflavona }\end{array}$ \\
\hline 95 & $\mathrm{H}$ & $\mathrm{OH}$ & $\mathrm{OCH}_{3}$ & $\mathrm{OH}$ & $\mathrm{H}$ & $\mathrm{OCH}_{3}$ & $\mathrm{OCH}_{3}$ & $\mathrm{H}$ & dalspinosina \\
\hline 96 & $\mathrm{H}$ & $\begin{array}{c}O-\beta \text {-D-apio- }(1 \rightarrow 6)- \\
\beta \text {-D-glc }\end{array}$ & $\mathrm{OCH}_{3}$ & $\mathrm{OH}$ & $\mathrm{H}$ & $\mathrm{OCH}_{3}$ & $\mathrm{OCH}_{3}$ & $\mathrm{H}$ & $\begin{array}{l}\text { dalspinosina } 7-O-\beta \text {-D-apio- } \\
(1 \rightarrow 6)-\beta-\mathrm{D} \text {-glc }\end{array}$ \\
\hline 97 & $\mathrm{H}$ & $\mathrm{OCH}_{3}$ & $\mathrm{OH}$ & $\mathrm{H}$ & $\mathrm{OCH}_{3}$ & $\mathrm{H}$ & $\mathrm{OCH}_{3}$ & $\mathrm{OCH}_{3}$ & $\begin{array}{c}\text { 6-hidroxi-7,2',4',5'-tetrametoxi- } \\
\text { isoflavona }\end{array}$ \\
\hline 98 & $\mathrm{H}$ & $\mathrm{OH}$ & $\mathrm{H}$ & $\mathrm{OH}$ & $\mathrm{OCH}_{3}$ & $\mathrm{OH}$ & $\mathrm{OCH}_{3}$ & $\mathrm{H}$ & khrinona $\mathrm{C}$ \\
\hline 99 & $\mathrm{H}$ & $\mathrm{OH}$ & $\mathrm{H}$ & $\mathrm{OH}$ & $\mathrm{OH}$ & $\mathrm{H}$ & $\mathrm{OCH}_{3}$ & $\mathrm{OH}$ & khrinona B \\
\hline 101 & $\mathrm{H}$ & $\mathrm{OH}$ & $\mathrm{OCH}_{3}$ & $\mathrm{H}$ & $\mathrm{OCH}_{3}$ & $\mathrm{OH}$ & $\mathrm{OCH}_{3}$ & $\mathrm{H}$ & pterosonina $\mathrm{C}$ \\
\hline 102 & $\mathrm{H}$ & $\mathrm{OH}$ & $\mathrm{OH}$ & $\mathrm{H}$ & $\mathrm{OCH}_{3}$ & $\mathrm{OH}$ & $\mathrm{OCH}_{3}$ & $\mathrm{H}$ & $\begin{array}{c}2^{\prime}, 4^{\prime} \text {-dimetoxi-3',6,7-trihidroxi- } \\
\text { isoflavona }\end{array}$ \\
\hline 103 & $\mathrm{H}$ & $\mathrm{OH}$ & $\begin{array}{l}O \text {-acetil- } \\
\beta \text {-D-glc }\end{array}$ & $\mathrm{OH}$ & $\mathrm{H}$ & $\mathrm{OH}$ & $\mathrm{OH}$ & $\mathrm{H}$ & 6- $(O$-acetil- $\beta$-D-glc)orobol \\
\hline 104 & $\mathrm{H}$ & $\mathrm{OH}$ & $\mathrm{OCH}_{3}$ & $\mathrm{H}$ & $\mathrm{OCH}_{3}$ & $\mathrm{H}$ & $\mathrm{OCH}_{3}$ & $\mathrm{OCH}_{3}$ & $\begin{array}{c}6,2^{\prime}, 4^{\prime}, 5^{\prime} \text {-tetrametoxi-7-hidroxi- } \\
\text { isoflavona }\end{array}$ \\
\hline 105 & $\mathrm{H}$ & $\begin{array}{c}O \text { - } \beta \text {-D-apio- }(1 \rightarrow 6)- \\
\beta \text {-D-glc }\end{array}$ & $\mathrm{OCH}_{3}$ & $\mathrm{H}$ & $\mathrm{OCH}_{3}$ & $\mathrm{H}$ & $\mathrm{OCH}_{3}$ & $\mathrm{OCH}_{3}$ & $\begin{array}{l}6,2^{\prime}, 4^{\prime}, 5^{\prime} \text {-tetrametoxi-7- } O-\beta \text {-D- } \\
\text { apio- }(1 \rightarrow 6)-\beta \text {-D-glc isoflavona }\end{array}$ \\
\hline 106 & $\mathrm{H}$ & $\mathrm{OH}$ & $\beta$-D-glc & $\mathrm{OH}$ & $\mathrm{H}$ & $\mathrm{OH}$ & $\mathrm{OH}$ & $\mathrm{H}$ & 6-( $\beta$-D-glc) orobol \\
\hline 108 & $\mathrm{H}$ & $\mathrm{OH}$ & $\mathrm{H}$ & $\mathrm{OH}$ & $\mathrm{OCH}_{3}$ & $\mathrm{H}$ & $\mathrm{OH}$ & $\mathrm{OH}$ & dalparvona B \\
\hline 109 & $\beta$-D-glc & $\mathrm{OH}$ & $\mathrm{H}$ & $\mathrm{OH}$ & $\mathrm{H}$ & $\mathrm{H}$ & $\mathrm{OH}$ & $\mathrm{OH}$ & orobol-8- $C$ - $\beta$-D-glc \\
\hline 110 & $\beta$-D-glc & $\mathrm{OH}$ & $\mathrm{H}$ & $\mathrm{OH}$ & $\mathrm{H}$ & $\mathrm{H}$ & $\mathrm{OH}$ & $\mathrm{OCH}_{3}$ & dalpanitina \\
\hline 111 & $\beta$-D-glc & $\mathrm{OH}$ & $\beta$-D-glc & $\mathrm{OH}$ & $\mathrm{H}$ & $\mathrm{H}$ & $\mathrm{OH}$ & $\mathrm{H}$ & paniculatina \\
\hline 112 & $\beta$-D-glc & $\mathrm{OH}$ & $\mathrm{H}$ & $\mathrm{OH}$ & $\mathrm{H}$ & $\mathrm{OH}$ & $\mathrm{OH}$ & $\mathrm{H}$ & mono- $C$-glc 8 -( $\beta$-D-glc)orobol \\
\hline 113 & $\mathrm{OCH}_{3}$ & $\mathrm{OH}$ & $\mathrm{H}$ & $\mathrm{H}$ & $\mathrm{OCH}_{3}$ & $\mathrm{OH}$ & $\mathrm{OCH}_{3}$ & $\mathrm{H}$ & $\begin{array}{l}7,3^{\prime} \text {-dihidroxi-8,2',4'-trimetoxi- } \\
\text { isoflavona }\end{array}$ \\
\hline 114 & $\mathrm{OH}$ & $\mathrm{OH}$ & $\mathrm{H}$ & $\mathrm{H}$ & $\mathrm{OCH}_{3}$ & $\mathrm{OH}$ & $\mathrm{OCH}_{3}$ & $\mathrm{H}$ & pterosonina A \\
\hline 115 & $\mathrm{OH}$ & $\mathrm{OCH}_{3}$ & $\mathrm{H}$ & $\mathrm{H}$ & $\mathrm{OCH}_{3}$ & $\mathrm{OH}$ & $\mathrm{OCH}_{3}$ & $\mathrm{H}$ & pterosonina B \\
\hline 116 & $\begin{array}{l}\text { 6"-O-acetil- } \beta \text { - } \\
\text { D-glc }\end{array}$ & $\mathrm{OH}$ & $\mathrm{H}$ & $\mathrm{OH}$ & $\mathrm{H}$ & $\mathrm{OH}$ & $\mathrm{OH}$ & $\mathrm{H}$ & 8-(6"- $O$-acetil- $\beta$-D-glc)orobol \\
\hline 117 & prenila & $O-\beta-\mathrm{D}-\mathrm{glc}$ & $\mathrm{H}$ & $\mathrm{OH}$ & $\mathrm{H}$ & $\mathrm{OH}$ & $\mathrm{OCH}_{3}$ & $\mathrm{H}$ & $\begin{array}{l}\text { 5,3'-dihidroxi-4'-metoxi-7-O- } \beta \text { - } \\
\text { glc-8-prenilisoflavona }\end{array}$ \\
\hline
\end{tabular}


Tabela 2. Estruturas das isoflavonas de 1-125 (cont.)

\begin{tabular}{|c|c|c|c|c|c|c|c|c|c|}
\hline $\mathrm{N}^{\circ}$ & $\mathrm{R}_{8}$ & $\mathrm{R}_{7}$ & $\mathrm{R}_{6}$ & $\mathrm{R}_{5}$ & $\mathrm{R}_{2}$, & $\mathrm{R}_{3}$, & $\mathrm{R}_{4}$, & $\mathrm{R}_{5}$, & Nome \\
\hline 118 & prenila & $\mathrm{OH}$ & $\mathrm{H}$ & $\mathrm{OH}$ & $\mathrm{H}$ & $\mathrm{OCH}_{3}$ & $\mathrm{OH}$ & $\mathrm{H}$ & $\begin{array}{l}\text { 5,7,4'-trihidroxi-3'-metoxi-8- } \\
\text { prenilisoflavona }\end{array}$ \\
\hline 119 & prenila & $\mathrm{OH}$ & $\mathrm{H}$ & $\mathrm{OH}$ & $\mathrm{H}$ & $\mathrm{OH}$ & $\mathrm{OCH}_{3}$ & $\mathrm{H}$ & $\begin{array}{l}5,7,3 \text { '-trihidroxi-4'-metoxi-8- } \\
\text { prenilisoflavona }\end{array}$ \\
\hline 120 & prenila & $\mathrm{OH}$ & prenila & $\mathrm{OH}$ & $\mathrm{H}$ & $\mathrm{H}$ & $\mathrm{OCH}_{3}$ & $\mathrm{H}$ & $\begin{array}{l}\text { 5,7-dihidroxi-4'-metoxi-6,8- } \\
\text { diprenilisoflavona }\end{array}$ \\
\hline 121 & $\beta$-D-glc & $\mathrm{OH}$ & $O-\beta$-D-glc & $\mathrm{OH}$ & $\mathrm{H}$ & $\mathrm{OH}$ & $\mathrm{OH}$ & $\mathrm{H}$ & $\begin{array}{c}\text { di- } C \text {-glicosidena- } 6,8-b i s(\beta-D-g l c) \\
\text { orobol }\end{array}$ \\
\hline 122 & $\mathrm{H}$ & $\mathrm{OH}$ & $\mathrm{OCH}_{3}$ & $\mathrm{OH}$ & $\mathrm{OCH}_{3}$ & $\mathrm{H}$ & $\mathrm{OCH}_{3}$ & $\mathrm{OCH}_{3}$ & caviunina \\
\hline 123 & $\mathrm{H}$ & $O-\beta-\mathrm{D}-\mathrm{glc}$ & $\mathrm{OCH}_{3}$ & $\mathrm{OH}$ & $\mathrm{OCH}_{3}$ & $\mathrm{H}$ & $\mathrm{OCH}_{3}$ & $\mathrm{OCH}_{3}$ & $\begin{array}{l}\text { caviunina 7-O- } \beta \text {-D- } \\
\text { glicopiranosídeo }\end{array}$ \\
\hline 124 & $\mathrm{H}$ & $\begin{array}{c}O-\beta \text {-D-apio- } \\
(1 " ' \rightarrow 6 ")-O-\beta \text {-D-glc }\end{array}$ & $\mathrm{OCH}_{3}$ & $\mathrm{OH}$ & $\mathrm{OCH}_{3}$ & $\mathrm{H}$ & $\mathrm{OCH}_{3}$ & $\mathrm{OCH}_{3}$ & dalsissoosídeo \\
\hline 125 & $\mathrm{OCH}_{3}$ & $\begin{array}{c}O-\beta-\mathrm{D} \text {-apio- }(1 \rightarrow 6)- \\
\beta-\mathrm{D}-\mathrm{glc}\end{array}$ & $\mathrm{H}$ & $\mathrm{OH}$ & $\mathrm{OCH}_{3}$ & $\mathrm{H}$ & $\mathrm{OCH}_{3}$ & $\mathrm{OCH}_{3}$ & $\begin{array}{l}\text { isocaviunina-7- } O-\beta \text {-D-apio- } \\
(1 \rightarrow 6)-\beta \text {-D-glicopiranosídeo }\end{array}$ \\
\hline
\end{tabular}

glc=glicosídeo; apio=apiofuranosídeo; rha=ramnopiranosídeo; gal=galactosídeo; xil=xilopiranosídeo; gen=gentiobiosídeo.

\section{Isoflavanonas}

As isoflavanonas $(\mathbf{1 2 6}$ a $\mathbf{1 5 5}, 12,5 \%)$ foram a segunda maior subclasse de isoflavonoides relatados em espécies da tribo Dalbergieae. Esses metabólitos também podem ser divididos em três grupos que compreendem compostos com padrões simples de substituição em oxigênios (Tabela 3), sendo 19 contendo hidroxi, metoxi e metilenodioxi, oito com substituinte prenila, um derivado glicosilado e dois compostos mistos (prenilados e glicosilados). A estrutura das isoflavanonas é caracterizada pela ausência de ligação dupla em C-2 do anel C, presentes nas isoflavonas (Tabela 3). ${ }^{6,18}$

Um total de 14 isoflavanonas $(46,6 \%)$ da tribo Dalbergieae são biologicamente ativas. Dessas, 12 foram isoladas na espécie $D$. parviflora e as atividades mais frequentes são estrogênica, antioxidante, citotóxica e antibacteriana.

A atividade antioxidante nos ensaios X/XO, ORAC e DPPH é relatada para as isoflavononas violanona (133), isolada de $D$. oliveri e D. parviflora, sativanona (129) e 3'-O-metilviolanona (135), obtidas de D. parviflora e D. odorifera, 7,3'-dihidroxi-4'-metoxi-isoflavanona (127), onogenina (131), dalparvina (134), dalparvina B (137), kenusanona G (138), secundiflorol H (142) e dalparvina C (145), isoladas apenas de D. parviflora. ${ }^{14}$ Os compostos 129, 133, 137, 138 e 145 apresentaram também atividade estrogência, assim como soforol (130), obtido de D. parviflora. $^{20}$

As isoflavanonas 3,5-dihidroxi-2',7-dimetoxi-2",2"-dimetilpirano[5",6":3',4']isoflavanona (151) e 3,4',5-trihidroxi-2',7-dimetoxi-3'-prenilisoflavanona (153), obtidas de D. melanoxylon, possuem elevada atividade antimicobacteriana frente a Mycobacterium tuberculosis. ${ }^{51} \mathrm{O}$ composto 153 apresentou $96 \%$ de inibição em ensaio in vitro e o estudo de docagem molecular demonstrou que ambos apresentam sítios ativos que favorecem essa atividade. ${ }^{51}$ Vestitona (128), isolada de D. odorifera, foi ativa frente a Ralstonia solanacearum, bactéria responsável pela murcha bacteriana. ${ }^{58}$ Esse composto apresentou ainda atividades antifúngica frente a Fusarium oxysporum e larvicida contra Aedes aegypti. .7,68 $^{-}$

Secundiflorol H (142), isolado de D. parviflora, e 3'-O-metilviolanona (135) apresentaram citotoxicidade frente às linhagens de células de carcionoma epidemoide de cavidade oral (KB), adenocarcinoide de mama (MCF-7) e câncer de pulmão de pequenas células (NCI-H187) e foram inativos frente a células não cancerígenas. ${ }^{78} \mathrm{~A}$ atividade inibitória da $\alpha$-glicosidase das isoflavononas 3'-O-metilviolanona (135) e sativanona (129) também é relatada. $^{22}$

\section{Pterocarpanos}

Os pterocarpanos constituem uma das maiores subclasses de isoflavonoides de Fabaceae, presentes em muitas espécies, além de serem reconhecidos como o segundo maior grupo de isoflavonoides naturais. ${ }^{142}$ Os pterocarpanos podem ser descritos como benzo-pirano-furano-benzenos que podem ser formados a partir de isoflavonas por acoplamento intramolecular na posição 4-cetona do anel B (Tabela 4). ${ }^{6,143}$

Os pterocarpanos são reconhecidos como fitoalexinas, compostos químicos de biossíntese induzida, que se acumulam em plantas, sob condições de estresse após infecção por microorganismos como bactérias e fungos. ${ }^{6}$ Os pterocarpanos são a terceira maior subclasse de compostos relatados na tribo Dalbergieae, representando 11,2\% (156 a 182, Tabela 4). Esses metabólitos apresentam uma gama de atividades biológicas. ${ }^{6}$ Um total de 11 pterocarpanos (40,7\%) da tribo Dalbergieae são biologicamente ativos. As atividades mais frequentes são citotóxica, antioxidante, anti-inflamatória e antimicrobiana.

O pterocarpano aracarpeno 2 (178), obtido de A. hypogaea, apresentou atividade antibacteriana. ${ }^{36}$ 3,8-dihidroxi-9-metoxipterocarpano (168), obtido de D. oliveri e de D. parviflora, apresentou atividade proliferativa de pelos e citotóxica frente às linhagens de células de carcionoma epidemoide de cavidade oral (KB), adenocarcinoide de mama (MCF-7) e câncer de pulmão de pequenas células (NCI-H187). ${ }^{71,78}$

Maackiaina (177), homopterocarpina (157), medicarpina (156), vesticarpano (165) e mucronucarpano (173) apresentaram atividades citotóxica frente a linhagens de células de câncer de mama (MCF7, T47d e HS578T), antimicrobiana, antiparasitária, anti-inflamatória, antioxidante e antimitótica. ${ }^{13,106,118-120}$

Homopterocarpina (157) exibiu ainda citotoxicidade em células de melanoma, cólon humano, mama e leucemia, atividade antifúngica contra Colletotrichum acutatum e Colletotrichum gloeosporioides e antiulcerogênica. ${ }^{120-122,124}$ Medicarpina (156) apresentou também atividade antifúngica, repelente contra zoósporo, larvicida contra Aedes aegypti e inibidora de agregação plaquetária. ${ }^{62,64,67}$

4-Hidroxi-3-metoxi-8,9-metilenodioxipterocarpano (179), obtido de $D$. parviflora, foi ativo frente às linhagens de células de carcionoma epidemoide de cavidade oral (KB), adenocarcinoide de mama (MCF-7) e câncer de pulmão de pequenas células (NCI-H187), ${ }^{78} \mathrm{en-}$ quanto 2,3,9-trimetoxipterocarpano (170), homopterocarpina (157) e vesticarpano (165), isolados de P. floribundum, apresentaram atividade citotóxica contra câncer de mama. ${ }^{118,120}$ Os pterocarpanos 165 e 170 
Tabela 3. Estruturas das isoflavanonas de 126-155<smiles>[R5]c1cc(C2([R3])COc3c([R8])c([R])c([R6])c([R5])c3C2=O)c([R1])c([R3])c1[R5]</smiles><smiles>CC(C)=CCC1Cc2c(cc(O)c3c2OC[C@@H](c2cc(O)c4c(c2)OCO4)C3=O)OC1(C)C</smiles>

140 dalhorridina<smiles>CC(C)/C=C/C(Cc1c(O)cc(O)c2c1OC[C@H](c1cc(O)c3c(c1)OCO3)C2=O)C(C)(C)O</smiles>

152 dalhorridinina

\begin{tabular}{|c|c|c|c|c|c|c|c|c|c|}
\hline $\mathrm{N}^{\circ}$ & $\mathrm{R}_{7}$ & $\mathrm{R}_{6}$ & $\mathrm{R}_{5}$ & $\mathrm{R}_{3}$ & $\mathrm{R}_{2}$, & $\mathrm{R}_{3}$, & $\mathrm{R}_{4}$ & $\mathrm{R}_{5}$, & Nome \\
\hline 126 & $\mathrm{OH}$ & $\mathrm{H}$ & $\mathrm{OH}$ & $\mathrm{H}$ & $\mathrm{H}$ & $\mathrm{H}$ & $\mathrm{OH}$ & $\mathrm{H}$ & dihidrogenisteína \\
\hline 127 & $\mathrm{OH}$ & $\mathrm{H}$ & $\mathrm{H}$ & $\mathrm{H}$ & $\mathrm{H}$ & $\mathrm{OH}$ & $\mathrm{OCH}_{3}$ & $\mathrm{H}$ & 7,3'-dihidroxi-4'-metoxi-isoflavanona \\
\hline 128 & $\mathrm{OH}$ & $\mathrm{H}$ & $\mathrm{H}$ & $\mathrm{H}$ & $\mathrm{OH}$ & $\mathrm{H}$ & $\mathrm{OCH}_{3}$ & $\mathrm{H}$ & vestitona \\
\hline 129 & $\mathrm{OH}$ & $\mathrm{H}$ & $\mathrm{H}$ & $\mathrm{H}$ & $\mathrm{OCH}_{3}$ & $\mathrm{H}$ & $\mathrm{OCH}_{3}$ & $\mathrm{H}$ & sativanona \\
\hline 130 & $\mathrm{OH}$ & $\mathrm{H}$ & $\mathrm{H}$ & $\mathrm{H}$ & $\mathrm{OH}$ & $\mathrm{H}$ & $\mathrm{O}-\mathrm{CH}_{2}-\mathrm{O}$ & & soforol \\
\hline 131 & $\mathrm{OH}$ & $\mathrm{H}$ & $\mathrm{H}$ & $\mathrm{H}$ & $\mathrm{OCH}_{3}$ & $\mathrm{H}$ & $\mathrm{O}-\mathrm{CH}_{2}-\mathrm{O}$ & & onogenina \\
\hline 132 & $\mathrm{OH}$ & $\mathrm{H}$ & $\mathrm{H}$ & $\mathrm{OH}$ & $\mathrm{OCH}_{3}$ & $\mathrm{H}$ & $\mathrm{OCH}_{3}$ & $\mathrm{H}$ & 2',4'-dimetoxi-3,7-dihidroxi-isoflavanona \\
\hline 133 & $\mathrm{OH}$ & $\mathrm{H}$ & $\mathrm{H}$ & $\mathrm{H}$ & $\mathrm{OCH}_{3}$ & $\mathrm{OH}$ & $\mathrm{OCH}_{3}$ & $\mathrm{H}$ & violanona \\
\hline 134 & $\mathrm{OH}$ & $\mathrm{H}$ & $\mathrm{H}$ & $\mathrm{H}$ & $\mathrm{OCH}_{3}$ & $\mathrm{H}$ & $\mathrm{OCH}_{3}$ & $\mathrm{OH}$ & dalparvina \\
\hline 135 & $\mathrm{OH}$ & $\mathrm{H}$ & $\mathrm{H}$ & $\mathrm{H}$ & $\mathrm{OCH}_{3}$ & $\mathrm{OCH}_{3}$ & $\mathrm{OCH}_{3}$ & $\mathrm{H}$ & 3'-O-metilviolanona \\
\hline 136 & $\mathrm{OH}$ & $\mathrm{H}$ & $\mathrm{H}$ & $\mathrm{H}$ & $\mathrm{OCH}_{3}$ & $\mathrm{H}$ & $\mathrm{OCH}_{3}$ & $\mathrm{OCH}_{3}$ & 2',4',5'-trimetoxi-7-hidroxi-isoflavanona \\
\hline 137 & $\mathrm{OH}$ & $\mathrm{H}$ & $\mathrm{H}$ & $\mathrm{H}$ & $\mathrm{OH}$ & $\mathrm{OCH}_{3}$ & $\mathrm{OCH}_{3}$ & $\mathrm{H}$ & dalparvina B \\
\hline 138 & $\mathrm{OH}$ & $\mathrm{H}$ & $\mathrm{OH}$ & $\mathrm{H}$ & $\mathrm{H}$ & $\mathrm{OH}$ & $\mathrm{OCH}_{3}$ & $\mathrm{H}$ & kenusanona $\mathrm{G}$ \\
\hline 139 & $\mathrm{OH}$ & $\mathrm{H}$ & $\mathrm{H}$ & $\mathrm{H}$ & $\mathrm{H}$ & $\mathrm{OCH}_{3}$ & $\mathrm{OH}$ & $\mathrm{OCH}_{3}$ & 7,4'-dihidroxi-3',5'-dimetoxi-isoflavanona \\
\hline 141 & $\mathrm{OCH}_{3}$ & $\mathrm{H}$ & $\mathrm{OH}$ & $\mathrm{OH}$ & $\mathrm{OH}$ & $\mathrm{H}$ & $\mathrm{OCH}_{3}$ & $\mathrm{H}$ & $2^{\prime}, 3^{\prime}, 7$-trihidroxi-4'-metoxi-isoflavanona \\
\hline 142 & $\mathrm{OH}$ & $\mathrm{H}$ & $\mathrm{OH}$ & $\mathrm{H}$ & $\mathrm{OCH}_{3}$ & $\mathrm{OH}$ & $\mathrm{OCH}_{3}$ & $\mathrm{H}$ & secundiflorol $\mathrm{H}$ \\
\hline 143 & $\mathrm{OH}$ & $\mathrm{H}$ & $\mathrm{H}$ & $\mathrm{OH}$ & $\mathrm{OH}$ & $\mathrm{OH}$ & $\mathrm{OCH}_{3}$ & $\mathrm{H}$ & isodarparvinol B \\
\hline 144 & $\mathrm{OH}$ & $\mathrm{H}$ & $\mathrm{OH}$ & $\mathrm{H}$ & $\mathrm{OCH}_{3}$ & $\mathrm{H}$ & $\mathrm{OH}$ & $\mathrm{OH}$ & dalparvina A \\
\hline 145 & $\mathrm{OH}$ & $\mathrm{H}$ & $\mathrm{H}$ & $\mathrm{OH}$ & $\mathrm{OH}$ & $\mathrm{OCH}_{3}$ & $\mathrm{OCH}_{3}$ & $\mathrm{H}$ & dalparvina $\mathrm{C}$ \\
\hline 146 & $\mathrm{OH}$ & $\mathrm{H}$ & $\mathrm{H}$ & $\mathrm{H}$ & $\mathrm{OH}$ & $\mathrm{OH}$ & $\mathrm{OCH}_{3}$ & prenila & $\begin{array}{l}\text { 7,2',3'-trihidroxi-4'-metoxi-5'- } \\
\text { prenilisoindanona }\end{array}$ \\
\hline 147 & $\mathrm{OH}$ & 4"-hidroxiprenila & $\mathrm{OH}$ & $\mathrm{H}$ & $\mathrm{OH}$ & $\mathrm{H}$ & $\mathrm{OH}$ & $\mathrm{H}$ & 4"-hidroxidifisolona \\
\hline 148 & $O$-glc & prenila & $\mathrm{OH}$ & $\mathrm{H}$ & $\mathrm{OH}$ & $\mathrm{H}$ & $\mathrm{OH}$ & $\mathrm{H}$ & 7-O-glicosildifisolona \\
\hline 149 & $O$-glc & prenila & $\mathrm{OH}$ & $\mathrm{H}$ & $\mathrm{OH}$ & $\mathrm{H}$ & O-glc & $\mathrm{H}$ & 7,4'-di- $O$-glicosildifisolona \\
\hline 150 & $\mathrm{OH}$ & $C$-glc & $\mathrm{OH}$ & $\mathrm{H}$ & $\mathrm{OH}$ & $\mathrm{H}$ & $\mathrm{OH}$ & $\mathrm{H}$ & macrocarposídeo \\
\hline 151 & $\mathrm{OCH}_{3}$ & $\mathrm{H}$ & $\mathrm{OH}$ & $\mathrm{OH}$ & $\mathrm{OCH}_{3}$ & $\begin{array}{c}\text { 2",2"-dimetilpirano } \\
{\left[5^{\prime \prime}, 6^{\prime \prime}: 33^{\prime}, 4^{\prime}\right]}\end{array}$ & & $\mathrm{H}$ & $\begin{array}{c}\text { 3,5-dihidroxi-2',7-dimetoxi-2",2"- } \\
\text { dimetilpirano [5",6":3',4'] isoflavanona }\end{array}$ \\
\hline 153 & $\mathrm{OCH}_{3}$ & $\mathrm{H}$ & $\mathrm{OH}$ & $\mathrm{OH}$ & $\mathrm{OCH}_{3}$ & prenila & $\mathrm{OH}$ & $\mathrm{H}$ & $\begin{array}{c}\text { 3,4',5-trihidroxi-2',7-dimetoxi } \\
\text {-3'-prenilisoflavanona }\end{array}$ \\
\hline 154 & $\mathrm{OH}$ & $\mathrm{H}$ & $\mathrm{OH}$ & $\mathrm{H}$ & $\mathrm{OH}$ & $\mathrm{OH}$ & $\mathrm{OCH}_{3}$ & prenila & $\begin{array}{c}5,7,2^{\prime}, 3^{\prime} \text {-tetrahidroxi-4'- metoxi-5' } \\
\text { prenilisoflavanona }\end{array}$ \\
\hline 155 & $\mathrm{OH}$ & $\mathrm{H}$ & $\mathrm{H}$ & $\mathrm{OH}$ & $\mathrm{OH}$ & $\mathrm{OH}$ & $\mathrm{OCH}_{3}$ & prenila & $\begin{array}{c}\text { 3,7,2',3'-tetrahidroxi-4'-metoxi-5'- } \\
\text { prenilisoflavanona }\end{array}$ \\
\hline
\end{tabular}

glc=glicosídeo.

também apresentaram atividade antimitótica. ${ }^{119}$ Metilnissolina (166), obtida de $D$. odorifera, apresentou atividade inibitória de agregação plaquetária, enquanto aeschinocarpina (182) e 2-metoximedicarpina (168), obtidos de A. fascicularis, apresentaram atividade citotóxica moderada e antiproliferativa frente às linhagens de células de carcinoma de próstata (DU-145) e adenocarcinoma de próstata (PC-3). ${ }^{25,64}$

\section{Rotenoides}

Os rotenoides da tribo Dalbergieae totalizaram 12 compostos.
Esses metabólitos (183 a 194, 5,0\%) constituem uma das subclasses de isoflavonoides mais importantes devido às suas propriedades inseticidas. São considerados derivados de isoflavonas pela incorporação de um átomo de carbono adicional, resultado da 2'-hidroxilação e subsequente metilação (2'-OMe) de uma isoflavona para formação do anel B do esqueleto rotenoide (Figura 2). Os rotenoides podem ser divididos em três subgrupos: desidrorotenoides (183-185), rotenoides $(\mathbf{1 8 6}, \mathbf{1 8 7}, \mathbf{1 8 9}, \mathbf{1 9 1 - 1 9 4})$ e 12a-hidroxirotenoides $\left(\mathbf{1 8 8}\right.$ e 190). ${ }^{6}$ Dentre os rotenoides da tribo Dalbergieae, somente três, amorfigenina (187), dalbinol (188) e amorfigenina- $\beta$-D-glicose (189), obtidos das 
Tabela 4. Estruturas dos pterocarpanos de 156-182

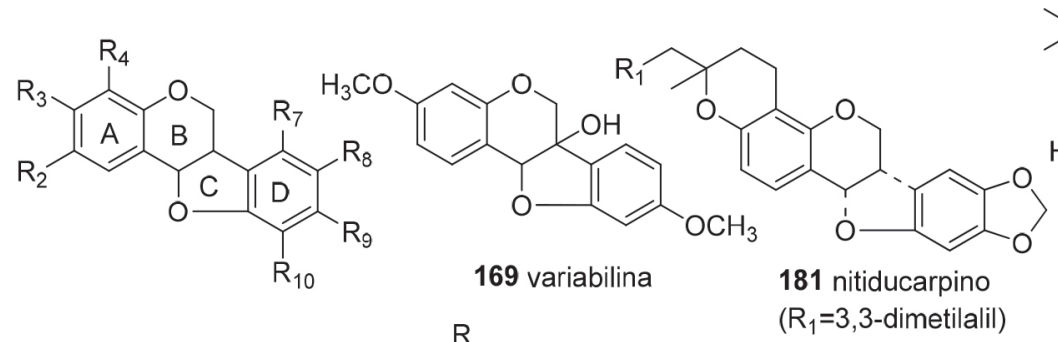

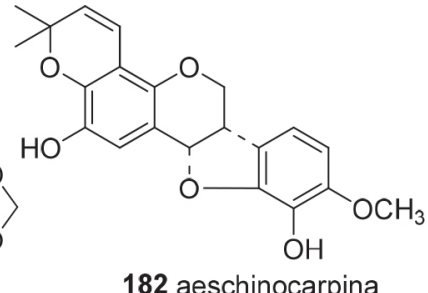<smiles>[R]c1cc2c(cc1O)OCC1c3ccc(OC)cc3OC21</smiles>

171 daljanelina $A$ 172 daljanelina B<smiles>[R]c1cc2c(cc1OC)OC1c3ccc(O)cc3OCC21</smiles>
164 daljanelina $\mathrm{C}$

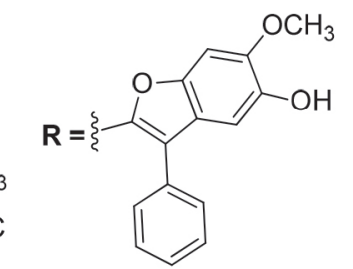

\begin{tabular}{|c|c|c|c|c|c|c|c|c|}
\hline $\mathrm{N}^{\circ}$ & $\mathrm{R}_{4}$ & $\mathrm{R}_{3}$ & $\mathrm{R}_{2}$ & $\mathrm{R}_{7}$ & $\mathrm{R}_{8}$ & $\mathrm{R}_{9}$ & $\mathrm{R}_{10}$ & Nome \\
\hline 156 & $\mathrm{H}$ & $\mathrm{OH}$ & $\mathrm{H}$ & $\mathrm{H}$ & $\mathrm{H}$ & $\mathrm{OCH}_{3}$ & $\mathrm{H}$ & medicarpina \\
\hline 157 & $\mathrm{H}$ & $\mathrm{OCH}_{3}$ & $\mathrm{H}$ & $\mathrm{H}$ & $\mathrm{H}$ & $\mathrm{OCH}_{3}$ & $\mathrm{H}$ & homopterocarpina \\
\hline 158 & $\mathrm{H}$ & $\mathrm{OH}$ & $\mathrm{H}$ & $\mathrm{H}$ & $\mathrm{O}-\mathrm{CH}_{2}-\mathrm{O}$ & & $\mathrm{H}$ & macckiaina \\
\hline 159 & $\mathrm{H}$ & $\mathrm{OCH}_{3}$ & $\mathrm{H}$ & $\mathrm{H}$ & $\mathrm{O}-\mathrm{CH}_{2}-\mathrm{O}$ & & $\mathrm{H}$ & pterocarpina \\
\hline 160 & $\mathrm{H}$ & $\mathrm{OH}$ & $\mathrm{H}$ & $\mathrm{H}$ & $\mathrm{OH}$ & $\mathrm{OCH}_{3}$ & $\mathrm{H}$ & 3,8-dihidroxi-9-metoxipterocarpano \\
\hline 161 & $\mathrm{H}$ & $\mathrm{OH}$ & $\mathrm{H}$ & $\mathrm{H}$ & $\mathrm{OCH}_{3}$ & $\mathrm{OH}$ & $\mathrm{H}$ & kushenina \\
\hline 162 & $\mathrm{H}$ & $\mathrm{OH}$ & $\mathrm{H}$ & $\mathrm{H}$ & $\mathrm{OCH}_{3}$ & $\mathrm{OCH}_{3}$ & $\mathrm{H}$ & 3-hidroxi-8,9-dimetoxipterocarpano \\
\hline 163 & $\mathrm{OH}$ & $\mathrm{OH}$ & $\mathrm{H}$ & $\mathrm{H}$ & $\mathrm{H}$ & $\mathrm{OCH}_{3}$ & $\mathrm{H}$ & 3,4-dihidroxi-9-metoxipterocarpano \\
\hline 165 & $\mathrm{H}$ & $\mathrm{OH}$ & $\mathrm{H}$ & $\mathrm{H}$ & $\mathrm{H}$ & $\mathrm{OCH}_{3}$ & $\mathrm{OH}$ & vesticarpano \\
\hline 166 & $\mathrm{H}$ & $\mathrm{OH}$ & $\mathrm{H}$ & $\mathrm{H}$ & $\mathrm{H}$ & $\mathrm{OCH}_{3}$ & $\mathrm{OCH}_{3}$ & metilnissolina \\
\hline 167 & $\mathrm{H}$ & $\mathrm{OCH}_{3}$ & $\mathrm{H}$ & $\mathrm{H}$ & $\mathrm{H}$ & $\mathrm{OH}$ & $\mathrm{OH}$ & 8,9-dihidroxi-3-metoxipterocarpano \\
\hline 168 & $\mathrm{H}$ & $\mathrm{OH}$ & $\mathrm{OCH}_{3}$ & $\mathrm{H}$ & $\mathrm{H}$ & $\mathrm{OCH}_{3}$ & $\mathrm{H}$ & 2-metoximedicarpina \\
\hline 170 & $\mathrm{H}$ & $\mathrm{OCH}_{3}$ & $\mathrm{OCH}_{3}$ & $\mathrm{H}$ & $\mathrm{H}$ & $\mathrm{OCH}_{3}$ & $\mathrm{H}$ & 2,3,9-trimetoxipterocarpano \\
\hline 173 & $\mathrm{H}$ & $\mathrm{OCH}_{3}$ & $\mathrm{OH}$ & $\mathrm{H}$ & $\mathrm{H}$ & $\mathrm{OCH}_{3}$ & $\mathrm{OH}$ & mucronucarpano \\
\hline 174 & $\mathrm{OH}$ & $\mathrm{OH}$ & $\mathrm{H}$ & $\mathrm{H}$ & $\mathrm{H}$ & $\mathrm{OCH}_{3}$ & $\mathrm{OH}$ & aracarpeno 1 \\
\hline 175 & $\mathrm{OH}$ & $\mathrm{OCH}_{3}$ & $\mathrm{H}$ & $\mathrm{H}$ & $\mathrm{H}$ & $\mathrm{OCH}_{3}$ & $\mathrm{OH}$ & melilotocarpano D \\
\hline 176 & $\mathrm{OH}$ & $\mathrm{OCH}_{3}$ & $\mathrm{H}$ & $\mathrm{H}$ & $\mathrm{H}$ & $\mathrm{OCH}_{3}$ & $\mathrm{OCH}_{3}$ & melilotocarpano C \\
\hline 177 & $\mathrm{OCH}_{3}$ & $\mathrm{OCH}_{3}$ & $\mathrm{H}$ & $\mathrm{H}$ & $\mathrm{H}$ & $\mathrm{OCH}_{3}$ & $\mathrm{OH}$ & odoricarpana \\
\hline 178 & $\mathrm{OH}$ & $\mathrm{OH}$ & $\mathrm{H}$ & $\mathrm{OH}$ & $\mathrm{H}$ & $\mathrm{OCH}_{3}$ & $\mathrm{H}$ & aracarpeno 2 \\
\hline 179 & $\mathrm{OH}$ & $\mathrm{OCH}_{3}$ & $\mathrm{H}$ & $\mathrm{H}$ & $\mathrm{O}-\mathrm{CH}_{2}-\mathrm{O}$ & & $\mathrm{H}$ & 4-hidroxi-3-metoxi-8,9-metilenodioxipterocarpano \\
\hline 180 & geranila & $\mathrm{OCH}_{3}$ & $\mathrm{H}$ & $\mathrm{H}$ & $\mathrm{O}-\mathrm{CH}_{2}-\mathrm{O}$ & & $\mathrm{H}$ & nutiducol \\
\hline
\end{tabular}

sementes de D. monetaria, são biologicamente ativos apresentando atividade larvicida. ${ }^{53}$

\section{Isoflavanas}

As isoflavanas da tribo Dalbergieae somaram 31 compostos (195-225, 13,0\%). Estes metabólitos compõem uma das menores subclasses de isoflavonoides nas quais incluem além de isoflavanas (195-220), isoflavanquinonas (221-223) e isoflavan-4-ois (224-225). As isoflavanas são reconhecidas como fitoalexinas e apresentam estruturas que diferem das isoflavononas pela ausência da carbonila no carbono 4 do anel C (Figura 3). As isoflavanquinonas diferem das isoflavanas pela presença de carbonilas nas posições 3' e 6' do anel B. Os isoflavan-4-ols se diferenciam das isoflavanonas pela presença de hidroxila no carbono 4 do anel $\mathrm{C}$ ao invés de carbonila (Tabela 5). Um total de 26 isoflavanas $(10,8 \%)$ foram relatadas na tribo Dalbergieae (Tabela 5), nas quais incluem um número significativo de estruturas com padrões simples de substituição no oxigênio (hidroxi, metoxi e metilenodioxi). ${ }^{6,18}$

Um total de 14 isoflavanas $(53,8 \%)$ da tribo Dalbergieae são biologicamente ativas. As atividades mais frequentes são antioxidante, antibacteriana, antifúngica, citotóxica e antimicrobiana.

As isoflavanas vestitol (195), obtida de D. parviflora, D. odorifera, D. tonkinensis e Machaerium ssp; sativano (198), 8-demetilduartina (205) e 3'-hidroxi-8-metoxivestitol (207), isoladas de D. parviflora, bem como duartina (208), obtida de D. parviflora e Machaerium ssp, apresentaram atividade antioxidante nos ensaios X/ XO, ORAC e DPPH. ${ }^{92,141}$ Vestitol (195) também apresentou atividades antibacteriana contra Ralstonia solanacearum, inibidora de agregação plaquetária e anti-hipercolesterêmica. ${ }^{58,64,65}$ Kotstrigoisoflavanol (224), obtido de K. strigosa, apresentou potencial antioxidante. ${ }^{105}$

Mucronulatol (200), obtido de D. oliveri, D. odorifera e D. parviflora, exibiu atividades antifúngica contra Fusarium oxysporum, citotóxica, antimalárica, antibacteriana, bem como anti-hipercolesterêmica e inibidora da $\alpha$-glicosidase. . $3,61,65,68^{2}$ '-Metoxivestitol (221), isolado de D. odorifera e D. oliveri e claussequinona (222), obtida de $D$. odorifera e $D$. candenatensis, apresentaram atividades anti-hipercolesterêmica, inibidora da $\alpha$-glicosidase e proliferativa de pelos. ${ }^{61,65,71} \mathrm{O}$ composto $\mathbf{2 2 2}$ apresentou ainda atividades antibacteriana e repelente contra zoósporos. ${ }^{41,62} 3$ '-Hidroxi-8-metoxivestitol 


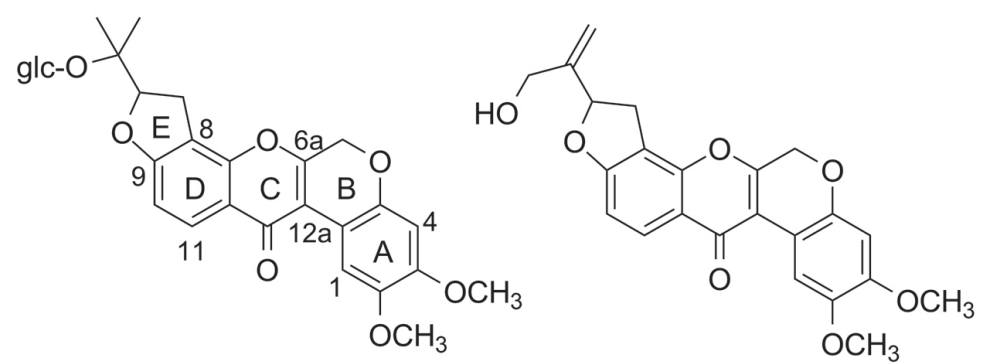

183 6'-glicosil-6a,12a-desidrorotenona
184 desidroamorphigenina

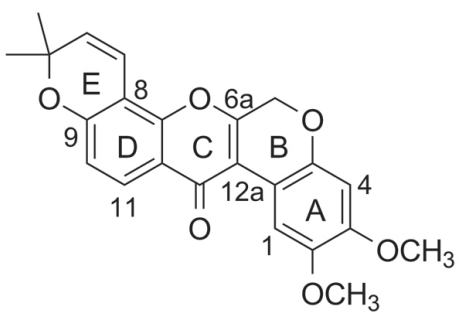

185 desidrodeguelina<smiles>[R10]CC(=C)[C@H]1Cc2c(ccc3c2O[C@@H]2COc4cc(OC)c(OC)cc4[C@@]2([R])C3=O)O1</smiles>

186 dalcochinina-8'-O- $\beta$-D-glicosídeo $\left(\mathrm{R}_{1}=\beta-\mathrm{D}-\mathrm{glicosil,} \mathrm{R}_{2}=\mathrm{H}\right)$

187 amorphigenina $\left(\mathrm{R}_{1}=\mathrm{H}, \mathrm{R}_{2}=\mathrm{H}\right)$

188 dalbinol $\left(\mathrm{R}_{1}=\mathrm{H}, \mathrm{R}_{2}=\mathrm{OH}\right)$

189 amorphigenina- $\beta$-D-glicose $\left(R_{1}=\beta\right.$-D-glicosil, $\left.R_{2}=H\right)$

190 dalbinol- $\beta$-D-glicose $\left(\mathrm{R}_{1}=\beta\right.$-D-glicosil, $\left.\mathrm{R}_{2}=\mathrm{OH}\right)$

193 dalpanol-O-glicosídeo $\left(\mathrm{R}_{1}=\beta-\mathrm{D}\right.$-glicosil, $\left.\mathrm{R}_{2}=\mathrm{H}\right)$

194 dalpanol $\left(\mathrm{R}_{1}=\mathrm{H}, \mathrm{R}_{2}=\mathrm{H}\right)$

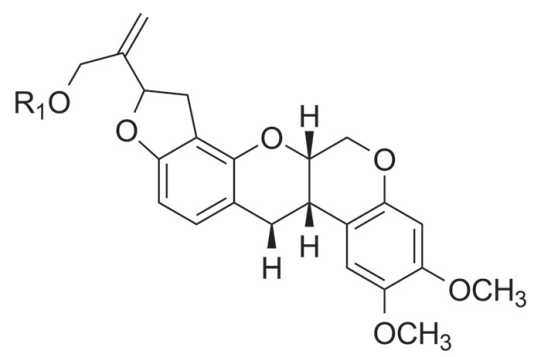

191 dihidrodalbinol $\left(\mathrm{R}_{1}=\mathrm{H}\right)$

192 dihidrodalbinol- $\beta$-D-glicose $\left(\mathrm{R}_{1}=\beta-\mathrm{D}-\mathrm{glicosil}\right)$

Figura 2. Fórmulas estruturais dos rotenoides de 183-194

(207), dalvelutinano B (214) e nitidulina (216), obtidos de D. velutina, foram avaliados quanto a citotoxicidade frente às linhagens celulares de carcinoma cervical humano (HeLa), carcinoma hepatocelular (HepG-2), adenocarcinoma de cólon (HT-29), carcinoma epidermoide de cavidade oral (KB), adenocarcinoide de mama (MCF-7), sendo que os compostos 207 e 214 foram moderadamente ativos contra três linhagens, enquanto o $\mathbf{2 1 6}$ demonstrou citotoxicidade significativa frente às cinco linhagens. ${ }^{97}$

Duartina (208) apresentou atividade citotóxica frente às linhagens de células carcinoma epidermoides de cavidade oral $(\mathrm{KB})$ e câncer de pulmão de pequenas células (NCI-H187). ${ }^{23} 4$ '- $O$-Metilpreglabridina (210) e 4'-O-metilglabridina (211), obtidos de D. robinioides, apresentaram atividade antimicrobiana contra Staphylococcus aureas, Mycobacterium smegmatis e Candida albicans, ${ }^{104}$ enquanto o secundiflorol G (217), obtido de A. fascicularis, apresentou atividade anticâncer significativa frente às linhagens de células de carcinoma de laringe (Hep-2), carcinoma epidermoide de cavidade oral (KB) e carcinoma cervical humano (HeLa). ${ }^{24,144}$

\section{Outros isoflavonoides}

Um total de 15 compostos (226-240, 6,2\%, Figura 4) não foram incluídos nas subclasses de isoflavonoides anteriormente discutidas e são classificados como isoflav-3-enos (226 e 227), pterocarpenos (228-232), cumestonas (233-235), arilbenzofurano-3-carbaldeído (236-238), cumaronocromona (239) e isoflavonoide benzofurano (240). Os isoflav-3-enos são similares às isoflavanas, acrescido de uma ligação dupla em C-3 do anel C. ${ }^{6}$

Os isoflav-3-enos tem um efeito promissor na inibição do NADH oxidase (tNOX) em células cancerígenas, o que por sua vez, leva à apoptose. Compostos dessa subclasse têm sido investigados contra cânceres de ovário, próstata e cervical. ${ }^{18}$ Entretanto, não há relato de atividade biológica para os isoflav-3-enos (226 e 227) da tribo Dalbergieae.

Os pterocarpenos apresentam estrutura derivada dos pterocarpanos com a presença de uma ligação dupla entre os carbonos 6a e 11a. Cumestonas são consideradas derivadas de isoflavonas, ${ }^{18}$ porém, alternativamente, também podem ser originadas de 2-hidroxi-3-arilcumarinas. ${ }^{6}$ As cumaronocromonas constituem uma pequena subclasse de isoflavonoides e sua biossíntese, não é clara, embora seja sugerido que hidroxi-isoflavonas, que às vezes co-ocorrem com as cumaronocromonas, podem ser os seus precursores. ${ }^{6,18}$ Não há um consenso sobre a origem biossintética de arilbenzofurano-3-carbaldeído. Macias et al. ${ }^{145}$ sugerem que sejam derivados de cumestanos pela abertura de um anel, entretanto, uma análise dos padrões de substituição indica que o anel A de 2-arilbenzofuranos é derivado do chiquimato, em vez do acetato, sugerindo uma via biossintética alternativa. ${ }^{6}$

Não foram encontrados relatos de atividades biológicas para os pterocarpenos (228-232), cumestonas (233-235) e cumaronocromona (239) da tribo Dalbergieae. Os três arilbenzofurano-3-carbaldeído (236-238), obtidos das folhas de $A$. inermis, apresentaram atividade antiplasmódica contra Plasmodium falciparum. ${ }^{31} \mathrm{O}$ isoflavonoide benzofurano (240), isolado da casca do caule de P. erinaceus, apresentou atividade antioxidante. ${ }^{123}$

\section{Considerações sobre as atividades biológicas da tribo Dalbergieae}

Os resultados da revisão das atividades biológicas dos isoflavonoides da tribo Dalbergieae mostraram um total de 81 compostos ativos, em 46 espécies pertencentes a 12 gêneros. Os gêneros mais estudados, do ponto de vista biológico, são Dalbergia, Machaerium, Andira e Pterocarpus com 21, 6, 6 e 4 espécies, respectivamente. Ribeiro et al. ${ }^{146}$ afirmam que investigações filogenéticas baseadas 


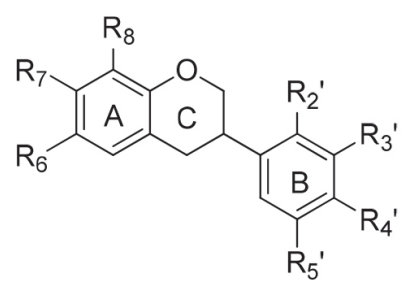<smiles>CCOc1ccc([C@H]2COc3c(ccc4c3C=CC(C)(C)O4)C2)c(O)c1</smiles><smiles>CC(C)=CCCC1(C)C=Cc2c(ccc3c2OC[C@H](C2=CC(=O)C(O)=CC2=O)C3)O1</smiles>

211 4'-O-metilglabridina 213 dalvelutinanes $A$

\begin{tabular}{|c|c|c|c|c|c|c|c|c|}
\hline & & 4 dalvel & anes $B$ & 0 & & $215 n$ & lano & 216 nitidulino \\
\hline $\mathrm{N}^{\circ}$ & $\mathrm{R}_{8}$ & $\mathrm{R}_{7}$ & $\mathrm{R}_{6}$ & $\mathrm{R}_{2}$ & $\mathrm{R}_{3}$. & $\mathrm{R}_{4}$ & $\mathrm{R}_{5}$, & Nome \\
\hline 195 & $\mathrm{H}$ & $\mathrm{OH}$ & $\mathrm{H}$ & $\mathrm{OH}$ & $\mathrm{H}$ & $\mathrm{OCH}_{3}$ & $\mathrm{H}$ & vestitol \\
\hline 196 & $\mathrm{H}$ & $\mathrm{OH}$ & $\mathrm{H}$ & $\mathrm{OCH}_{3}$ & $\mathrm{H}$ & $\mathrm{OH}$ & $\mathrm{H}$ & neovestitol \\
\hline 197 & $\mathrm{H}$ & $\mathrm{OCH}_{3}$ & $\mathrm{H}$ & $\mathrm{OH}$ & $\mathrm{H}$ & $\mathrm{OCH}_{3}$ & $\mathrm{H}$ & 7-O-metilvestitol \\
\hline 198 & $\mathrm{H}$ & $\mathrm{OH}$ & $\mathrm{H}$ & $\mathrm{OCH}_{3}$ & $\mathrm{H}$ & $\mathrm{OCH}_{3}$ & $\mathrm{H}$ & sativano \\
\hline 199 & geranila & $\mathrm{OH}$ & $\mathrm{H}$ & $\mathrm{H}$ & $\mathrm{H}$ & $\mathrm{OCH}_{3}$ & $\mathrm{H}$ & candenatenina $\mathrm{F}$ \\
\hline 200 & $\mathrm{H}$ & $\mathrm{OH}$ & $\mathrm{H}$ & $\mathrm{OH}$ & $\mathrm{OCH}_{3}$ & $\mathrm{OCH}_{3}$ & $\mathrm{H}$ & mucronulatol \\
\hline 201 & $\mathrm{H}$ & $\mathrm{OH}$ & $\mathrm{H}$ & $\mathrm{OH}$ & $\mathrm{H}$ & $\mathrm{OCH}_{3}$ & $\mathrm{OCH}_{3}$ & 5'-metoxivestitol \\
\hline 202 & $\mathrm{H}$ & $\mathrm{OH}$ & $\mathrm{H}$ & $\mathrm{OH}$ & $\mathrm{OH}$ & $\mathrm{OCH}_{3}$ & $\mathrm{H}$ & 7,2',3'-trihidroxi-4'-metoxi-isoflavana \\
\hline 203 & $\mathrm{OH}$ & $\mathrm{OH}$ & $\mathrm{H}$ & $\mathrm{OCH}_{3}$ & $\mathrm{H}$ & $\mathrm{OCH}_{3}$ & $\mathrm{OCH}_{3}$ & 7,8-dihidroxi-2', $4^{\prime}, 5^{\prime}$-trimetoxi-isoflavana \\
\hline 204 & $\mathrm{OH}$ & $\mathrm{OH}$ & $\mathrm{H}$ & $\mathrm{OH}$ & $\mathrm{OCH}_{3}$ & $\mathrm{OCH}_{3}$ & $\mathrm{H}$ & 7,8,2'-trihidroxi-3',4'-dimetoxi-isoflavana \\
\hline 205 & $\mathrm{OH}$ & $\mathrm{OH}$ & $\mathrm{H}$ & $\mathrm{OCH}_{3}$ & $\mathrm{OH}$ & $\mathrm{OCH}_{3}$ & $\mathrm{H}$ & 8-demetilduartina \\
\hline 206 & $\mathrm{OH}$ & $\mathrm{OH}$ & $\mathrm{H}$ & $\mathrm{OH}$ & $\mathrm{OH}$ & $\mathrm{OCH}_{3}$ & $\mathrm{H}$ & 3',8-dihidroxivestitol \\
\hline 207 & $\mathrm{OCH}_{3}$ & $\mathrm{OH}$ & $\mathrm{H}$ & $\mathrm{OH}$ & $\mathrm{OH}$ & $\mathrm{OCH}_{3}$ & $\mathrm{H}$ & 3'-hidroxi-8-metoxivestitol \\
\hline 208 & $\mathrm{OCH}_{3}$ & $\mathrm{OH}$ & $\mathrm{H}$ & $\mathrm{OCH}_{3}$ & $\mathrm{OH}$ & $\mathrm{OCH}_{3}$ & $\mathrm{H}$ & duartina \\
\hline 209 & $\mathrm{OCH}_{3}$ & $\mathrm{OH}$ & $\mathrm{H}$ & $\mathrm{OH}$ & $\mathrm{OCH}_{3}$ & $\mathrm{OCH}_{3}$ & $\mathrm{H}$ & isoduartina \\
\hline 210 & prenila & $\mathrm{OH}$ & $\mathrm{H}$ & $\mathrm{OH}$ & $\mathrm{H}$ & $\mathrm{OCH}_{3}$ & $\mathrm{H}$ & 4'-O-metilpreglabridina \\
\hline 212 & $\mathrm{H}$ & $\mathrm{OH}$ & $\mathrm{OH}$ & $\mathrm{OCH}_{3}$ & $\mathrm{OH}$ & $\mathrm{OCH}_{3}$ & $\mathrm{H}$ & 6,7,3'-trihidroxi-2',4'-dimetoxi-isoflavana \\
\hline 217 & $\mathrm{H}$ & $\mathrm{OH}$ & $\mathrm{H}$ & $\mathrm{OH}$ & $\mathrm{OH}$ & $\mathrm{OCH}_{3}$ & 2-metilbut-3-en-2-ila & secundifforol G \\
\hline 218 & $\mathrm{OCH}_{3}$ & $\mathrm{OCH}_{3}$ & $\mathrm{OH}$ & $\mathrm{OH}$ & $\mathrm{OCH}_{3}$ & $\mathrm{OCH}_{3}$ & $\mathrm{H}$ & machaerol B \\
\hline 219 & $\mathrm{OH}$ & $\mathrm{OCH}_{3}$ & $\mathrm{OH}$ & $\mathrm{OH}$ & $\mathrm{OCH}_{3}$ & $\mathrm{OCH}_{3}$ & $\mathrm{H}$ & machaerol C \\
\hline
\end{tabular}<smiles>CCC(=O)CC1(O)C=C(C2COc3cc(O)ccc3C2)C(=O)C=C1OC</smiles>

220 pteroyanina $J$<smiles>[R2]C1=CC(=O)C([C@H]2COc3c(ccc(O)c3[R7])C2)=CC1=O</smiles>

221 mucroquinona $\left(\mathrm{R}_{1}=\mathrm{R}_{2}=\mathrm{OCH}_{3}\right)$

222 claussequinona $\left(\mathrm{R}_{1}=\mathrm{H}, \mathrm{R}_{2}=\mathrm{OCH}_{3}\right)$

223 7,4'-dihidroxi-isoflavanquinona $\left(\mathrm{R}_{1}=\mathrm{H}, \mathrm{R}_{2}=\mathrm{OH}\right)$<smiles>[R]c1ccc(C2COc3cc([R])c([R])cc3C2[R3])c(O)c1</smiles>

224 kotstrigoisoflavanol $\left(\mathrm{R}_{1}, \mathrm{R}_{2}=\mathrm{OCH}_{2} \mathrm{O}, \mathrm{R}_{3}=\mathrm{OH}, \mathrm{R}_{4}=\mathrm{OCH}_{3}\right)$

225 bolusantol $D$ $\left(\mathrm{R}_{1}=\mathrm{R}_{3}=\mathrm{OH}, \mathrm{R}_{2}=\mathrm{H}, \mathrm{R}_{4}=\mathrm{OCH}_{3}\right)$

Figura 3. Fórmulas estruturais de isoflavana (220), isoflavanquinonas (221-223) e isoflavan-4-ols (224-225)

em dados moleculares mostraram uma relação íntima entre os gêneros Dalbergia e Machaerium os quais pertencem ao mesmo clado (Dalbergia), o que pode explicar suas similaridades em composição química e atividades biológicas. Entretanto, estes estudos apontaram ainda, uma proximidade entre os gêneros Machaerium e Aeschynomene seção Ochopodium, ${ }^{9,146}$ no entanto, os resultados não são conclusivos e não há indicativo de similaridade a partir dos dados quimiossistemáticos. Os isoflavonoides de duas espécies do gênero Dalbergia (D. parviflora e D. odorifera) apresentaram uma grande variedade de atividades biológicas, com destaque para atividade estrogênica, sobretudo atribuída às isoflavonas, relatadas em $D$. parviflora.

A maioria dos resultados de atividades biológicas foi obtida a partir de ensaios com compostos isolados. Dentre as 46 espécies com atividades biológicas apenas A. sensitiva, A. fluminensis, D. 
<smiles>[R]c1cc(OC)c([R])c([R])c1C1=Cc2ccc(O)c([R])c2OC1</smiles>

226 7,2'-dihidroxi-8,4'-dimetoxi-isoflavena $\left(\mathrm{R}_{1}=\mathrm{OCH}_{3}, \mathrm{R}_{2}=\mathrm{OH}, \mathrm{R}_{3}=\mathrm{R}_{4}=\mathrm{H}\right)$ 227 odoriflavena $\left(\mathrm{R}_{1}=\mathrm{R}_{2}=\mathrm{H}, \mathrm{R}_{3}=\mathrm{OCH}_{3}, \mathrm{R}_{4}=\mathrm{OH}\right)$<smiles>[R]c1cc2c(c([R])c1OC)OCc1c-2oc2c([R])c(OC)ccc12</smiles>

228 bryacarpeno $1\left(\mathrm{R}_{1}=\mathrm{R}_{3}=\mathrm{OH}, \mathrm{R}_{2}=\mathrm{OCH}_{3}\right)$ 229 bryacarpeno $2\left(\mathrm{R}_{1}=\mathrm{H}, \mathrm{R}_{2}=\mathrm{OCH}_{3}, \mathrm{R}_{3}=\mathrm{OH}\right)$ 230 bryacarpeno $3\left(\mathrm{R}_{1}=\mathrm{H}, \mathrm{R}_{2}=\mathrm{R}_{3}=\mathrm{OCH}_{3}\right)$ 231 bryacarpeno $4\left(\mathrm{R}_{1}=\mathrm{OH}, \mathrm{R}_{2}=\mathrm{H}, \mathrm{R}_{3}=\mathrm{OCH}_{3}\right)$ 232 bryacarpeno $5\left(\mathrm{R}_{1}=\mathrm{R}_{2}=\mathrm{H}, \mathrm{R}_{3}=\mathrm{OCH}_{3}\right)$<smiles>[R]c1cc2oc3c4ccc(O)c([R])c4oc(=O)c3c2cc1[R]</smiles>

233 dalbergestano $\left(\mathrm{R}_{1}=\mathrm{OCH}_{3}, \mathrm{R}_{2}=\mathrm{R}_{3}=\mathrm{OH}\right)$

234 12-O-metilcomestrol $\left(\mathrm{R}_{1}=\mathrm{R}_{2}=\mathrm{H}, \mathrm{R}_{3}=\mathrm{OCH}_{3}\right)$ 235 medicagol $\left(\mathrm{R}_{1}=\mathrm{H}, \mathrm{R}_{2}, \mathrm{R}_{3}=\mathrm{OCH}_{2} \mathrm{O}\right)$

Figura 4. Fórmulas estruturais de outros isoflavonoides (226-240)

odorifera e $V$. guianensis apresentaram resultados exclusivamente com extrato bruto ou frações. ${ }^{26,27,65,136-138}$ As atividades predominantes nos isoflavonoides foram antimicrobiana (13 espécies), estrogênica (11 espécies), citotóxica (11 espécies) e antioxidante (9 espécies). As isoflavonas $(\mathbf{1 - 1 2 5}, 52,1 \%)$ compõem a subclasse de isoflavonoides com maior número e variedade de atividades biológicas, seguidas pelas isoflavanonas (126-155, 12,5\%), pterocarpanos (156-182, $11,2 \%)$ e isoflavanas (195-220, 10,8\%). As atividades biológicas mais frequentes entre as isoflavonas foram citotóxica, estrogênica, antioxidante, osteogênica e anti-helmíntica. Esses resultados demonstram o potencial biológico da tribo Dalbergieae, bem como sugerem a necessidade de mais estudos visando a descoberta de princípios ativos, uma vez que apenas $33,75 \%$ dos compostos relatados apresentaram alguma atividade biológica.

\section{CONSIDERAÇÕES FINAIS}

A revisão bibliográfica dos isoflavonoides da tribo Dalbergieae (Fabaceae-Papilionoideae), no período de 1945 a 2019, mostrou a ocorrência de 240 compostos distribuídos em 69 espécies pertencentes a 15 gêneros. Os isoflavonoides glicosilados totalizaram 48 compostos que corresponde a $20 \%$. Formononetina (2), biochanina A (17) e medicarpina (156) são os isoflavonoides que ocorrem com maior frequência na tribo. Os gêneros que apresentaram maior número de espécies com ocorrência de isoflavonoides foram Dalbergia, Machaerium, Andira e Pterocarpus, com 31, 9, 6 e 5, respectivamente.<smiles>[R6]c1ccc(-c2oc3cc([R])c([R])c(O)c3c2C)c([R])c1[R]</smiles>

236 andinermal $A\left(\mathrm{R}_{1}=\mathrm{H}, \mathrm{R}_{2}=\mathrm{R}_{3}=\mathrm{R}_{5}=\mathrm{OCH}_{3}, \mathrm{R}_{4}=\mathrm{OH}\right)$

237 andinermal $B\left(\mathrm{R}_{1}, \mathrm{R}_{2}=\mathrm{OCH}_{2} \mathrm{O}, \mathrm{R}_{3}=\mathrm{R}_{5}=\mathrm{OCH}_{3}, \mathrm{R}_{4}=\mathrm{OH}\right)$

238 andinermal $\mathrm{C}\left(\mathrm{R}_{1}=\mathrm{H}, \mathrm{R}_{2}=\mathrm{R}_{3}=\mathrm{OCH}_{3}, \mathrm{R}_{4}=\mathrm{R}_{5}=\mathrm{OH}\right)$<smiles>COc1cc(O)c2c(=O)c3c(oc2c1)oc1cc(OC)c(O)cc13</smiles>

239 dalbergicromona

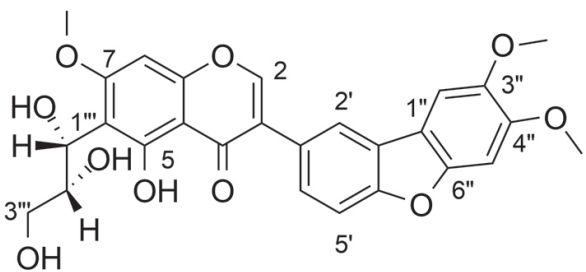

240 3-(7,8-dimetoxi[b,d]furan-2-il)-5-hidroxi-7-metoxi-6((1R,2R)-1,2,3-trihidroxipropil)-4H-cromen-4-ona

A tribo Dalbergieae demonstrou possuir um importante potencial biológico com 46 espécies e 81 isoflavonoides bioativos, no entanto, representa apenas $33,75 \%$ dos compostos isolados e identificados, sugerindo a necessidade de mais estudos para verificar o potencial biológico dos demais compostos e espécies.

\section{AGRADECIMENTOS}

Os autores agradecem a Capes, CNPq (406126/2018-6 e 302470/2018-2) e CNPq/INCTBioNat (465637/2014-0) pelo apoio financeiro e bolsa.

\section{REFERÊNCIAS}

1. Sousa, E. A.; Chaves, M. H.; Rev. Virtual Quim. 2019, 11, 1767.; Wink, M.; Botschen, F.; Gosmann, C.; Schafer, H.; Waterman, P. G.; Annu. Plant Rev. 2010, 40, 364.

2. Singh, R.; Geetanjali. Em Natural Products and Drug Discovery: An Integrated Approach; Mandal, S. C.; Mandal, V.; Konishi, T., eds.; Elsevier-Copyright: Amsterdam, 2018, cap. 6.

3. Reynolds, T.; Phytochemistry 2007, 68, 2887.

4. Zidorn, C.; Phytochemistry 2019, 163,147.

5. Pereira, R.; Souza, E. B.; Fontenelle, R. O. S.; Vasconcelos, M. A.; Santos, H. S.; Teixeira, E. H. Hoehnea 2019, 46, 1; Rocha e Silva, H.; Silva, C. C. M.; Caland-Neto, L. B.; Lopes, J. A. D.; Citó, A. M. G. L.; Chaves, M. H. Quim. Nova 2007, 30, 1877; Zaruchi, J. L.; 
Phytochemical Dictionary of the Leguminosae, Chapman \& Hall London, 1994.

6. Veitch N.; Nat. Prod. Rep. 2013, 30, 988; Veitch N.; Nat. Prod. Rep. 2009, 26, 776.; Veitch N.; Nat. Prod. Rep. 2007, 24, 417.

7. LPWG (Legume Phylogeny Working Group); Taxon 2017, 66, 44.

8. Sharma, V.; Ramawat, K. G. Em Natural Products; Ramawat, K. G., Mérillon, J. M., eds.; Springer-Verlag: Berlin Heidelberg, 2013, cap. 60.

9. Wojciechowski, M. F.; Lavin, M.; Sanderson, M. J.; Am. J. Bot. 2004, 91, 1846; Lavin, M.; Pennington, R. T.; Klitgaard, B. B.; Sprent, J. I.; Lima, H. C.; Gasson, P. E. Am. J. Bot. 2001, 88, 503.

10. Lewis, G. P.; Schrire, B. D.; Mackinder, B. A.; Lock, J. M. Legumes of the World, Royal Botanic Gardens, Kew: London, 2005.

11. Forzza, R. C; Baumgratz, J. F. A.; Bicudo, C. E. M.; Carvalho Júnior, A. A.; Costa, A.; Costa. D. P.; Hopkins, M., Leitman, P. M.; Lohmann, L. G.; Maia, L. C.; Martinelli, G.; Menezes, M.; Morim, M. P.; Coelho, M. A. N.; Peixoto, A. L.; Pirani, J. R.; Prado, J.; Queiroz, L. P.; Souza, V. C.; Stehmann, J. R.; Sylvestre, L. S.; Walter, B. M. T.; Zappi, D.; Catálogo de plantas e fungos do Brasil, Instituto de Pesquisas Jardim Botânico do Rio de Janeiro: Rio de Janeiro, 2010, v. 2.

12. Hegnauer, R.; Barkmeijer, R. J. G.; Phytochemistry 1993, 34, 3.

13. Amen, Y. M.; Marzouk, A. M.; Zaghloul, M. G.; Afifi, M. S.; Nat. Prod. Res. 2015, 29,1388.

14. Judd, W. S.; Campbell, C. S.; Kellog, E. A.; Stevens, P. F.; Plant Systematics: a phylogenetic approach. Sinauer Associates. Sunderland: Massachusetts, 2007.

15. Souza, V. C.; Lorenzi, H. Botânica Sistemática: Guia Ilustrado para Identificação das Famílias de Angiospermas da Flora Brasileira, baseado em APG II, Plantarum: Nova Od, 2008.

16. Wojciechowski, M. F. Em Advances in Legume Systematics; Klitgaard, B. B., Bruneau, A. eds.; Royal Botanic Gardens, Kew: London, 2003; Klitgaard, B. B.; Lavin, M. Em Legumes of the World; Lewis, G. P., Schirire, B., Mackinder, B., Lock, M., eds.; The Royal Botanical Gardens, Kew: London, 2005.

17. Ferreira, J. J. S.; Oliveira, A. C. S.; Queiroz, R. T.; Silva, J. S.; Rodriguésia 2019, 70, e03502017.

18. Dewick, P. M.; Medicinal natural products: a biosynthetic approach. 3. ed. New York: John Wiley \& Sons, 2009, 539 p.

19. Reynaud, J., Guilet, D.; Terreux, R.; Lussignol, M.; Walchshofer, N.; Nat. Prod. Res. 2005, 22, 504

20. Umehara, K.; Nemoto, K.; Kimuima K.; Matsushita, A.; Terada, E.; Monthakantirat, O.; Eknamkul, W.; Miyase, T.; Warashina, T.; Degawa, M.; Noguchi, H.; Phytochemistry 2008, 69, 546. 2008; Umehara, K.; Nemoto, K.; Matsushita, A.; Terada, E.; Monthakantirat, O.; Eknamkul, W.; Miyase, T.; Warashina, T.; Degawa, M.; Noguchi, H.; J. Nat. Prod. 2009, 72, 2163.

21. Araujo, J. M. E.; Melo, L. S. M.; Araujo, E. D.; Fernandes, R. P. M.; Scher, R.; Braz. Arch. Biol. Technol. 2018, 61, e18160461.

22. Zhao, C.; Liu, Y.; Cong, D.; Zhang, H.; Yu, J.; Jiang, Y.; Cui, X.; Sun, J.; Biomed. Chromatogr. 2013, 27, 1621.

23. Songsiang, U.; Wanich, S.; Pitchuanchom, S.; Netsopa, S.; Uanporn, K.; Yenjai, C.; Fitoterapia 2009, 80, 427.

24. Caamal-Fuentes, E. E; Peraza-Sánchez, S. R.; Torres-Tapia, L. W.; MooPuc, R. E.; Molecules 2015, 20, 13563.

25. Caamal-Fuentes, E.; Moo-Puc, R.; Torres-Tapia, L. W.; Peraza-Sanchez, S. R.; Nat. Prod. Commun. 2013, 8, 1421.

26. Ignoato, M. C.; Fabrão, R. M.; Schuquel, I. T. A.; Botelho, M. F. P.; Santin, S. M. O.; Quim. Nova 2012, 35, 2241

27. Arruda, R. F.; Alves-Olher, V. G.; Vandresen, F.; Schuquel, I. T. A.; Bersani-Amado, C. A.; Nakamura, C. V.; Silva, C. C.; Rev. Virtual Quim. 2018, 10, 698

28. Silva, V. C.; Carvalho, M. G.; Borba, H. R.; Silva, S. L. C.; Rev. Bras. Farmacogn. 2008, 18, 573.
29. Silva, V. C.; Alves, A. N.; Santana, A.; Carvalho, M. G.; Quim Nova 2006, 29, 1184.

30. Garcez, W. S.; Garcez, F. R.; Tieppo, C.; Freitas, O.N.; Hamerski, L.; Biochem. Syst. Ecol. 2010, 38, 1242.

31. Kraft, C.; Jenett-Siems, K.; Siems, K.; Solis, P. N.; Gupta, M. P.; Bienzle, U.; Eich, E.; Phytochemistry 2001, 58, 769.

32. Kraft, C.; Jenett-Siems, K.; Siems, K.; Gupta, M. P.; Bienzle, U.; Eich, E.; J. Ethnopharmacol. 2000, 73, 131.

33. Silva, B. P.; Velozo, L. S. M.; Parente, J. P.; Fitoterapia 2000, 71, 663.

34. Garcia, M. G.; Nascimento, C. C.; Ferreira, A. G.; Lima, M. P.; Chem. Nat. Compd. 2018, 54, 856.

35. Almeida, J. G. L.; Silveira, E. R.; Pessoa, O. D. L.; Magn. Reson. Chem. 2008, 46, 103

36. Sobolev, V. S.; Neff, S. A.; Gloer, J. B.; Shabana, I.; Khan, S. I.; Tabanca, N.; Lucca, A. J.; Wedge, D. E.; Phytochemistry 2010, 71, 2099.

37. Hausen, B. M.; Bruhn G.; Koenig, W. A. Contact Dermatitis 1991, 25, 149; Ferreira, M. A.; Moir, M.; Thomson, R. H.; J. Chem. Soc. 1974, 1, 2429; Ferreira, M. A.; Moir, M.; Thomson, R. H.; J. Chem. Soc. 1975, 1,1113 .

38. Araujo, C. A. C.; Alegrio, L. V.; Leon, L. L.; Phytochemistry 1998, 49, 751

39. Alegrio, L. V.; Braz-Filho, R.; Gottlieb, O. R.; Phytochemistry 1989, 28, 2359.

40. Abdou, J. P.; Momeni, J.; Adhikari, A.; Tsabang, N.; Tchinda, A. T.; Choudhary, M. I.; Nkengfack, A. E.; Phytochem. Lett. 2017, 21, 109.

41. Hamburger, M. O.; Cordell, G. A.; J. Nat. Prod. 1987, 50, 696

42. Cheenpracha, S.; Karalai, C.; Ponglimanont, C.; Kanjana-Opas, A.; J. Nat. Prod. 2009, 72, 1395.

43. Svasti, J.; Srisomsap, C.; Techasakul, S.; Surarit, R.; Phytochemistry 1999, 50, 739

44. Rao, P. R.; Narayanan, M. C.; Gopalakrishnan, S. M.; Shanmugam, N. N.; J. Asian Nat. Prod. Res. 2006, 8, 143.

45. Ramesh, P.; Yuvahajan, C. R.; J. Nat. Prod. 1995, 58, 1240.

46. Piccinelli, A. L.; Lotti, C.; Campone, L.; Cuesta-Rubio, O.; Fernandez, M. C.; Rastrelli, L.; J. Agric. Food Chem. 2011, 59, 6484.

47. Donnelly, D. M. X.; Keenan, P. J.; Prendergast, J. P.; Phytochemistry 1973, 12, 1157

48. Khan, I. A.; Avery, M. A.; Burandt, C. L.; Goins, D. K.; Mikell, J. R.; Nash, T. E.; Azadegan, A.; Walker, L. A.; J. Nat. Prod. 2000, 63, 1414.

49. Narayanan, M. C.; Rao, P. R.; Shanmugam, N. N.; Gopalakrishnan, S. M.; Devi, K.; Nat. Prod. Res. 2007, 10, 903.

50. Malhotra, A.; Murti, V. V. S.; Seshadri, T. R.; Tetrahedron 1966, 23, 405.

51. Mutai, P.; Heydenreich, M.; Thoithi, G.; Mugumbate, G.; Chibale, K.; Yenesew, A.;

Phytochem. Lett. 2013, 6, 671.

52. Nunes, D. S.; Haag, A.; Bestmann, H. J.; Eur. J. Org. Chem. 1989, 4, 331.

53. Abe, F.; Donnelly, D. M X.; Morett, C.; Polonsky, J.; Phytochemistry 1985, 24, 1071

54. Mathias, L.; Vieira, I. J. C.; Braz-Filho, R.; Rodrigues-Filho, E. A.; J. Nat. Prod. 1998, 61, 1158.

55. Bekker, M.; Malan, E.; Steenkamp, J. A.; Brandt, E. V.; Phytochemistry 2002, 59,415

56. Ferreira, J. A.; Nel, J. W.; Brandt, E. V.; Bezuidenhoudt, B. C. B.; Ferreira, D. J.; Chem. Soc. Perkin Trans. I 1995, 26, 1049.

57. Li, L.; Liu, J. Z.; Luo, M. Wang, W.; Huang, Y. Y.; Efferth, T.; Wang, H. M.; Fu, Y. J.; J. Chromatogr. B 2016, 1033-1034, 40.

58. Zhao, X.; Mei, W.; Gong, M.; Zuo, W.; Bai, H.; Dai, H.; Molecules 2011, 16, 9775

59. Lee, C.; Lee, J. W.; Jin, Q.; Jang, D. S.; Lee, S. J.; Lee, D.; Hong, J. T.; Kim, Y.; Lee, M. K.; Hwang, B. Y.; Bioorg. Med. Chem. Lett. 2013, 23, 4263. 
60. Zhang, D. Y.; Zu, Y. G.; Fu, Y. J.; Luo, M.; Gu, C. B.; Wang, W.; Yao, X. H.; Sep. Purif. Technol. 2011, 83, 91.

61. Choi, C. W.; Choi, Y. H.; Cha, M. R.; Yoo, D. S.; Kim, Y. S.; Yon, G. H.; Hong, K. S.; Kim, Y. H.; Ryu, S. Y.; J. Agric. Food Chem. 2010, 58, 9988.

62. Islam, T. M.; Z. Naturforsch. 2008, 63, 233.

63. Liu, R. X.; Wang, Q.; Guo, H. Z.; Li, L.; Bi, K. S.; Guo, D. A.; J. Pharm. Biomed. Anal. 2005, 39, 469.; Liu, R. X.; Ye, M.; Guo, H. Z.; Bi, K. S.; Guo, D. A.; Rapid Commun. Mass Spectrom. 2005, 19, 1557.

64. Goda, Y., Katayama, M., Ichikawa, K., Shibuya, M., Kiuchi, F., Sankawa, U.; Chem. Pharm. Bull. 1985, 33, 5606.

65. Yahara, S.; Saijo, R.; Nohara, T.; Konishi, R.; Yamahara, J.; Kawasaki, T.; Miyahara, K.; Chem. Pharm. Bull. 1985, 33, 5130.

66. Yahara, S.; Ogata, T.; Saijo, R.; Konishi, R.; Yamahara, J.; Miyahara, K.; Nohara, T.; Chem. Pharm. Bull. 1989, 37, 979.

67. Pluempanupat, S.; Kumrungsee, N.; Pluempanupat, W.; Ngamkitpinyo, K.; Chavasiri, W.; Bullangpoti, V.; Koul, O.; Ind. Crops Prod. 2013, 44, 653.

68. Deesamer, S.; Kokpol, U.; Chavasiri, W.; Douillard, S.; Peyrot, V.; Vidal, N.; Combes, S.; Finet, J.; Tetrahedron 2007, 63, 12986.

69. Ito, C; Itoigawa, M.; Kanematsu, T.; Ruangrungsi, N.; Mukainaka, T.; Tokuda, H.; Nishino, H.; Furukawa, H.; Phytochemistry 2003, 64, 1265.

70. Donnelly, D. M. X.; Kavanach, P. J.; Phytochemistry 1974, 13, 2587.

71. Park, S. J., Nhiem, N. X., Tai, B. H., Anh, H. L. T., Oh, S. H., Sung, J. H., Van Kiem, P.; Nat. Prod. Commun. 2017, 12, 1729.

72. Parthasarathy, M. R.; Seshadri, T. R.; Varma, R. S.; Phytochemistry 1976, 15, 1025.

73. Adinarayana, D.; Rao, J. R.; Tetrahedron 1972, 28, 5377.

74. Parthasarathy, M. R.; Seshadri, T. R.; Varma, R. S.; Letters to the Editor 1974, 43, 74.

75. Radhakiushniah, M. Phytochemistry 1973, 12, 3003.

76. Ibrahim, S. Bandi, A. K. R.; Gunasekar, D.; Murthy, M. M.; Rao, T. P.; Blond, A.; Bodo, B.; Nat. Prod. Commun. 2007, 2, 1109.

77. Castellano, G.; Torrens, F.; Int. J. Mol. Sci. 2015, 16, 12891.

78. Songsiang, U.; Hahnvajanawong, C.; Yenjai, C. Fitoterapia 2011, 82, 1169 .

79. Monthakantirat, O.; Umehara, K.; Matsushita, A.; Terada, E.; De -Eknamkul, W.; Miyase, T.; Warashina, T.; Noguchi, H.; Planta Med. 2007, 73, 379 .

80. Braz Filho, R.; Almeida, M. E. L.; Gottlieb, O. R.; Phytochemistry 1973, $12,1187$.

81. Charles, J. A.; Gandhidasan, R.; Indian J. Chem. 2006, 45B, 1282.

82. Dixit, P.; Chillara, R.; Khedgikar, V.; Gautam, J.; Kushwaha, P.; Kumar, A.; Singh, D.; Trivedi, R.; Maurya, R.; Bioorg. Med. Chem. Lett. 2012, 22,890 .

83. Reddy, R. V. N.; Reddy, N. P.; Khalivulla, S. I.; Reddy, M. V. B.; Gunasekar, D.; Blond, A.; Bodo, B.; Phytochem. Lett. 1 2008, 1, 23.

84. Farag, S. F.; Ahmed, A. S.; Terashima, K.; Takaya, Y.; Niwa, M.; Phytochemistry 2001, 57, 1263.

85. Sarg, T.; Ateya, A. M.; Ghani, A. A.; Badr, W.; Hams, G.; Pharm. Biol. 1999, 37,54 .

86. Kavimani, S.; Ilango, R.; Krishnamoorthy, G.; Tamizhmozhi, M.; Jaykar, B.; Nagarajan, N. S.; Manoj, C.; Indian J. Heterocycl. Chem. 1997, 6, 235 .

87. Kumar, S.; Steiner, T.; Subramanian, K.; J. Chem. Crystallogr. 1999, 29 , 99.

88. Narayanan, V.; Nagarajan, N. S.; Phytochemistry 1988, 27, 2364.

89. Radha, R.; Vasantha, V. S.; Pitchumani, K.; Nat. Prod. Commun. 2015, 10, 1959 .

90. Cook, J. T.; Olli, W. D.; Sutherland, I. O.; Gottlieb, O. R.; Phytochemistry 1978, 17, 1419.

91. Nagarajan, N. S.; Sethuraman, M. G.; Manoj, C. N.; Rao, R. P.; Nat. Prod. Res. 2006, 20, 195.
92. Son, N. T.; Kamiji, M.; Huong, T. T.; Kubo, M.; Cuong, N. M.; Fukuyama, Y.; Med. Chem. Res. 2019, 28, 1441.

93. Son, N. T.; Oda, M.; Hayashi, N.; Yamaguchi, D.; Kawagishi, Y.; Takahashi, F.; Harada, K.; Cuong, N.; Fukuyama, Y.; Nat. Prod. Commun. 2018, 13, 157.

94. Innocent, E.; Magadula, J. J.; Kihampa, C.; Heydenreich, M.; Nat. Prod. Commun. 2010, 5, 903.

95. Kurosawa, K.; Ollis, W. D.; Suthwland, I. O.; Gottlieb, O. R.; Phytochemistry 1978, 17, 1417.

96. Kaennakam, S.; Sukandar, E. R.; Rassamee, K.; Siripong, P.; Tip-Pyang, S.; Phytochem. Lett. 2019, 31, 187.

97. Kaennakam, S.; Siripong, P.; Tip-Pyang, S.; J. Nat. Med. 2017, 71, 310.

98. Kaennakam, S.; Siripong, P.; Tip-Pyang, S. Nat. Prod. Res. 2015, 30, 1493.

99. Loan, P. T.; Le Anh, H. T.; Cuc, N. T.; Yen, D.T.; Hang, D. T.; Ha, T. M.; Nhiem, N. X.; Van Du, N.; Thai, T. H.; Van Minh, C.; Van Kiem, P.; Nat. Prod. Commun. 2014, 9, 809

100. Chawla, H.; Chibber, S. S.; Seshadri, T. R.; Phytochemistry 1974, 13, 2301.

101. Kurosawa, K.; Ollis, W. D.; Redman, B. T.; Sutherland, I. O.; Alves, H. M.; Gottlieb, O. R.; Phytochemistry 1978, 17, 1423.

102. Jurd, L.; Stevens, K.; Manners, G. Phytochemistry 1972, 11, 2535.

103. Castro, O.; Lopez, J.; Vergara, A.; J. Nat. Prod. 1986, 49, 680.

104. Vila, J.; Balderrama, L.; Bravo, J. L.; Almanza, G.; Codina, C.; Bastida, Connolly, J.; Phytochemistry 1998, 49, 2525.

105. Awouafack, M. D.; Tchuenguem, R. T.; Ito, T.; Dzoyem, J. P.; Tane, P.; Morita, H.; Helv. Chim. Acta 2016, 99, 321.

106. Seo, E. K.; Kim, N. C.; Mi, Q.; Chai, H.; Wall, M. E.; Wani, M. C.; Navarro, H. A.; Burgess, J. P.; Graham, J. G.; Cabieses, F.; Tan, G. T.; Farnsworth, N. R.; Pezzuto, J. M.; Kinghorn, A. D.; J. Nat. Prod. 2001, 64,1483 .

107. Elsohly, H. N.; Joshi A. S., Nimrod, A. C.; Planta Med. 1999, 65, 490.

108. Carvalho, A. A.; Santos, L. R.; Sousa, R. P.; Freitas, J. S.; Araújo, B. Q.; Chaves, M. H. Em Ciências Biológicas Campo Promissor em Pesquisa 2; Freitas, R. M., eds.; Atena Editora: Ponta Grossa, 2019, cap. 13.

109. Ollis, W. D.; Redman, B. T.; Sutherland, I. O.; Gottlieb, O. R.; Magalhães, M. T.; Phytochemistry 1978, 17, 1383.

110. Kurosawa, K.; Ollis, W. D.; Suthwland, I. O.; Gottlieb, O. R.; Oliveira, A. B.; Phytochemistry 1978, 17, 1405.

111. Ollis, W. D.; Sutherland, I. O.; Alves, H. M.; Gottlieb, O. R.; Phytochemistry 1978, 17, 1401.

112. Ogiyama, K.; Yasue, M.; Phytochemistry 1973, 12, 2544.

113. Kurosawa, K.; Ollis, W. D.; Redman, B. T.; Suthwland, I. O.; Gottlieb, O. R.; Phytochemistry 1978, 17, 1413.

114. Adem, F. A.; Mbaveng, A. T.; Kuete, V.; Heydenreich, M.; Ndakala, A.; Irungu, B.; Yenesew, A.; Efferth, T.; Phytomedicine 2019, 58, 152853.

115. Xu, Y. J.; Foubert, K.; Dhooghe, L.; Lemière, F.; Maregesi, S.; Coleman, C. M.; Zou, Y.; Ferreira, D.; Apers, S.; Pieters, L.; Phytochemistry 2012, 79, 121.

116. Dhooghe, L.; Maregesi, S.; Mincheva, I.; Ferreira, D.; Marais, J. P J.; Lemière, F.; Matheeussen, A.; Cos, P.; Maes, L.; Vlietinck, A.; Apers, S.; Pieters, L.; Phytochemistry 2010, 71, 785.

117. Selvam, C.; Jordan, B. C.; Prakash, S.; Mutisya, D.; Thilagavathi, R.; Eur. J. Med. Chem. 2017, 128, 219.

118. Militão, G. C. G.; Prado, M. P.; Pessoa, C.; Moraes, M. O.; Silveira, E. R.; Lima, M. A. S.; Veloso, P. A.; Costa-Lotufo, L. V.; Machado-Santelli, G. M.; Biochimie 2014, 104, 147.

119. Militão, G. C.; Jimenez, P. C.; Wilke, D. V.; Pessoa, C.; Falcão, M. J.; Lima, M. A. S.; Silveira, E. R.; Moraes, M. O.; Costa-Lotufo, L. V.; Planta Med. 2005, 71, 683.

120. Falcão, M. J. C.; Pouliquem, Y. B. M.; Lima, M. A. S.; Gramosa, N. V.; Costa-Lotufo, L. V.; militão, G. C. G.; Pessoa, C.; Moraes, M. O.; Silveira, E. R.; J. Nat. Prod. 2005, 68, 423. 
121. Martinez, J.; García, C.; Durango, D.; Bol. Latinoam. Caribe Plant. Med. Aromat. 2017, 16, 14-25.

122. Reyes-Chilpa, R.; Gómez-Garibay, F.; Moreno-Torres, G.; JiménezEstrada, M.; Quiroz-Vásquez, R. I.; Holzforschung 1998, 52, 459.

123. Toukam, P. D.; Tagatsing, M. F.; Yamthe, L. R. T.; Baishya, G.; Barua, N. C.; Tchinda, A. T.; Mbafor, J. T.; Phytochem. Lett. 2018, 28, 69.

124. Olaleye, M. T.; Akinmoladun, A. C.; Crown, O. O.; Ahonsi, K. E.; Adetuyi, A. O.; Asian Pac. J. Trop. Med. 2013, 200.

125. Tip-Pyang, S.; Aree, T.; Sichaem, J.; Chem. Nat. Compd. 2019, 55, 121.

126. Cooke, R. G; Rae, I. D.; Aust. J. Chem. 1964, 17, 379.

127. Verma, K. S.; Jam, A. K.; Nagar, A.; Gupta, S. R.; Planta Med. 1986, 4, 315.

128. Anandharajan, R.; Pathmanathan, K.; Shankernarayanan, N. P.; Vishwakarma, R. A.; Balakrishnan, A.; J. Ethnopharmacol. 2005, 97, 253.

129. Mitra, J.; Joshi, T.; Phytochemistry 1983, 22, 2326.

130. Mitra, J.; Joshi, T.; Phytochemistry 1982, 21, 2429.

131. Krishnaveni, K. S.; Rao, J. V. S.; Chem. Pharm. Bull. 2000, 48, 1373.

132. Krishnaveni, K. S.; Rao, J. V. S.; Phytochemistry 2000, 53, 605.

133. Krishnaveni, K. S.; Rao, J. V. S.; J. Asian Nat. Prod. Res. 2000, 2, 219.

134. Su, Z.; Wang, P.; Yuan, W.; Li, S.; Nat. Prod. Commun. 2014, 9, 1483.

135. Su, Z.; Wang, P.; Yuan, W.; Li, S.; Planta Med. 2013, 79, 487.

136. Souza, R. F.; Silva, G. A.; Arruda, A. C.; Silva, M. N.; Santos, A. S.; Grisólia, D. P. A.; Silva, M. B.; Salgado, C. G.; Arruda, M. S. P.; J. Braz. Chem. Soc. 2017, 28, 1132.
137. Souza, R. F.; Silva, J. K. R.; Silva, G. A.; Arruda, A. C.; Silva, M. N.; Arruda, M. S. P.; Rev. Virtual Quim. 2015, 7, 1893.

138. Souza, R. F.; Marinho, V. H. S.; Silva, G. A.; Costa Jr., L. M.; Silva, J. K. R.; Bastos, G. N. T.; Arruda, A. C.; Silva, M. N.; Arruda, M. S. P.; J. Braz. Chem. Soc. 2013, 24, 1857.

139. Nascimento, Y. M.; Abreu, L. S.; Lima, R. L.; Silva, A. D. S.; Costa, V. C. O; Melo, J. I. M.; Scotti, M. T.; Sobral, M. V.; Araujo, S. S.; Filho, M. A. G.; Silva, M. S.; Tavares, J. F.; Rev. Bras. Farmacogn. 2018, 28 , 192.

140. Mariana, A.; Marinela, P.; Rev. Roum. Chim. 2007, 52, 537.

141. Promden, W.; Monthakantirat, O.; Umehara, K.; Noguchi, H.; DeEknamkul, W.; Molecules 2014, 19, 2226.

142. Jimenez, L.; Alvarez, M.; Munoz, M.; Rodriguez, R.; Phytochem. Rev. 2008, 7, 125.

143. Pistelli, L.; Noccioli, C.; Appendino, G.; Bianchi, F.; Sterner, O.; Ballero, M.; Phytochemistry 2003, 64, 595.

144. Castillo-Bautista, C. M.; Torres-Tapia, L. W.; Lagunas-Martínez, A.; Contreras-Ochoa, C. O.; Peraza-Sanchez, S. R.; Moo-Puc, R.; Nat. Prod. Res. 2019, 16, 1.

145. Macias, F. A.; Simonet, A. M.; Galindo, J. C. G.; Castellano, D.; Phytochemistry 1999, 50, 35.

146. Ribeiro, R. A.; Lavin, M.; Lemos-Filho, J. P.; Mendonça Filho, C. V.; Santos, F. R.; Lovato, M. B.; Syst. Bot. 2007, 32, 762. 